\title{
ON THE STRANGE DUALITY CONJECTURE FOR ABELIAN SURFACES
}

\author{
ALINA MARIAN AND DRAGOS OPREA
}

\begin{abstract}
We study Le Potier's strange duality conjecture for moduli spaces of sheaves over generic abelian surfaces. We prove the isomorphism for abelian surfaces which are products of elliptic curves, when the moduli spaces consist of sheaves of equal ranks and fiber degree 1 . The birational type of the moduli space of sheaves is also investigated. Generalizations to arbitrary product elliptic surfaces are given.
\end{abstract}

\section{INTRODUCTION}

There are three versions of Le Potier's strange duality conjecture for abelian surfaces, formulated and supported numerically in MO2. In this article, we confirm two of them for product abelian surfaces. This establishes the conjecture over an open subset in the moduli space of polarized abelian surfaces.

1.1. The strange duality morphism. To set the stage, let $(X, H)$ be a polarized complex abelian surface. For a coherent sheaf $V \rightarrow X$, denote by

$$
v=\operatorname{ch} V \in H^{\star}(X, \mathbb{Z})
$$

its Mukai vector. Fix two Mukai vectors $v$ and $w$ such that the orthogonality condition

$$
\chi(v \cdot w)=0
$$

holds. We consider the two moduli spaces $\mathfrak{M}_{v}^{+}$and $\mathfrak{M}_{w}^{+}$of $H$-semistable sheaves of type $v$ and $w$ with fixed determinant. They carry two determinant line bundles

$$
\Theta_{w} \rightarrow \mathfrak{M}_{v}^{+}, \quad \Theta_{v} \rightarrow \mathfrak{M}_{w}^{+}
$$

whose global sections we seek to relate.

More precisely, according to Le Potier's strange duality conjecture [LP] [MO2], if the condition

$$
c_{1}(v \cdot w) \cdot H>0
$$

is satisfied, the jumping locus

$$
\Theta_{v w}^{+}=\left\{(V, W): h^{1}(V \otimes W) \neq 0\right\} \subset \mathfrak{M}_{v}^{+} \times \mathfrak{M}_{w}^{+}
$$


is expected to be a divisor. Further, its defining equation is a section of the split bundle

$$
\Theta_{w} \otimes \Theta_{v} \rightarrow \mathfrak{M}_{v}^{+} \times \mathfrak{M}_{w}^{+}
$$

conjecturally inducing an isomorphism

$$
\mathrm{D}^{+}: H^{0}\left(\mathfrak{M}_{v}^{+}, \Theta_{w}\right)^{\vee} \rightarrow H^{0}\left(\mathfrak{M}_{w}^{+}, \Theta_{v}\right) .
$$

This expectation is supported numerically: it was established in [MO2] that

$$
\chi\left(\mathfrak{M}_{v}^{+}, \Theta_{w}\right)=\chi\left(\mathfrak{M}_{w}^{+}, \Theta_{v}\right)=\frac{\chi\left(X, L^{+}\right)}{d_{v}+d_{w}}\left(\begin{array}{c}
d_{v}+d_{w} \\
d_{v}
\end{array}\right)
$$

where $L^{+}$is a line bundle on $X$ with

$$
c_{1}\left(L^{+}\right)=c_{1}(v \cdot w)
$$

and

$$
d_{v}=\frac{1}{2} \operatorname{dim} \mathfrak{M}_{v}^{+}, \quad d_{w}=\frac{1}{2} \operatorname{dim} \mathfrak{M}_{w}^{+}
$$

Similarly, letting $\mathfrak{M}_{v}^{-}$and $\mathfrak{M}_{w}^{-}$denote the moduli spaces of sheaves with fixed determinant of their Fourier-Mukai transforms, we have the symmetry

$$
\chi\left(\mathfrak{M}_{v}^{-}, \Theta_{w}\right)=\chi\left(\mathfrak{M}_{w}^{-}, \Theta_{v}\right)=\frac{\chi\left(X, L^{-}\right)}{d_{v}+d_{w}}\left(\begin{array}{c}
d_{v}+d_{w} \\
d_{v}
\end{array}\right)
$$

where $L^{-}$is a line bundle on $X$ with

$$
c_{1}\left(L^{-}\right)=c_{1}(\widehat{v} \cdot \widehat{w})
$$

the hats denoting the Fourier-Mukai transforms. The map

$$
\mathrm{D}^{-}: H^{0}\left(\mathfrak{M}_{v}^{-}, \Theta_{w}\right)^{\vee} \rightarrow H^{0}\left(\mathfrak{M}_{w}^{-}, \Theta_{v}\right)
$$

induced by the theta divisor $\Theta_{v w}^{-} \subset \mathfrak{M}_{v}^{-} \times \mathfrak{M}_{w}^{-}$is expected to be an isomorphism.

1.2. Results. We will establish the isomorphisms for abelian surfaces which split as products of elliptic curves

$$
X=B \times F .
$$

We regard $X$ as a trivial fibration $\pi_{B}: X \rightarrow B$, and write $\sigma$ and $f$ for the class of the zero section and of the fiber over zero. We assume that the polarization $H$ is suitable in the sense of $[\mathrm{F}]$ i.e.

$$
H=\sigma+N f, \text { for } N \gg 0 .
$$

Over simply connected elliptic surfaces, for coprime rank and fiber degree, the moduli space of sheaves is birational to the Hilbert scheme of points, as shown by Bridgeland B]. For sheaves with fixed determinant, the situation is subtler over elliptic abelian 
surfaces. A refinement of Bridgeland's argument, using a Fourier-Mukai transform with kernel given by a universal Atiyah bundle, allows us to prove the following result.

Theorem 1. Let $X=B \times F$ be a product abelian surface, and let $v$ be a Mukai vector such that the rank $r$ and the fiber degree $d=c_{1}(v) \cdot f$ are coprime. Then, the moduli space $\mathfrak{M}_{v}^{+}$is birational to

$$
\mathfrak{X}_{v}^{+}=\left\{(Z, b): a_{B}(Z)=r b\right\} \subset X^{\left[d_{v}\right]} \times B,
$$

where $a_{B}$ denotes the addition over the base elliptic curve $B$.

A similar statement holds for the moduli space $\mathfrak{M}_{v}^{-}$. By contrast, the generalized Kummer variety $K_{v}$ associated to the higher rank vectors is birational to the Kummer variety in rank 1, as noted in $[\mathrm{Y}]$. This fact is recovered in two ways while establishing Theorem 1,

The following two theorems capture our main results concerning strange duality.

Theorem 2. Let $X=B \times F$ be a product abelian surface. Let $v$ and $w$ be two orthogonal Mukai vectors of equal ranks $r \geq 3$, with

$$
c_{1}(v) \cdot f=c_{1}(w) \cdot f=1 .
$$

Then,

$$
\mathrm{D}^{+}: H^{0}\left(\mathfrak{M}_{v}^{+}, \Theta_{w}\right)^{\vee} \rightarrow H^{0}\left(\mathfrak{M}_{w}^{+}, \Theta_{v}\right)
$$

is an isomorphism.

Similarly, we show

Theorem 3. Let $X=B \times F$ be a product abelian surface. Assume $v$ and $w$ are two orthogonal Mukai vectors of ranks $r, s \geq 3$ and equal Euler characteristics $\chi=\chi^{\prime}$, with

$$
c_{1}(v) \cdot f=c_{1}(w) \cdot f=1 .
$$

Then,

$$
\mathrm{D}^{-}: H^{0}\left(\mathfrak{M}_{v}^{-}, \Theta_{w}\right)^{\vee} \rightarrow H^{0}\left(\mathfrak{M}_{w}^{-}, \Theta_{v}\right)
$$

is an isomorphism.

In particular, the theorems imply that $\Theta^{ \pm}$are divisors in the products $\mathfrak{M}_{v}^{ \pm} \times \mathfrak{M}_{w}^{ \pm}$.

1.2.1. Higher genus. The requirement that $B$ be elliptic can in fact be removed in Theorem 2. Indeed, consider $C$ a smooth projective curve of genus $g \geq 1$, and let $\sigma$ denote the zero section of the trivial fibration

$$
X=C \times F \rightarrow C .
$$

We show 
Theorem 2A. Assume that $X=C \times F$ is a product surface as above, and let $v, w$ be orthogonal Mukai vectors of equal ranks $r \geq g$ and $r \neq 2$, such that

(i) the determinants are fixed of the form

$$
\operatorname{det} v=\mathcal{O}(\sigma) \otimes \ell_{v}, \quad \operatorname{det} w=\mathcal{O}(\sigma) \otimes \ell_{w},
$$

for generic line bundles $\ell_{v}$ and $\ell_{w}$ of fixed degree over the curve $C$;

(ii) $\operatorname{dim} \mathfrak{M}_{v}^{+}+\operatorname{dim} \mathfrak{M}_{w}^{+} \geq 8 r(g-1)$.

Then

$$
\mathrm{D}^{+}: H^{0}\left(\mathfrak{M}_{v}^{+}, \Theta_{w}\right)^{\vee} \rightarrow H^{0}\left(\mathfrak{M}_{w}^{+}, \Theta_{v}\right)
$$

is an isomorphism.

1.3. Comparison. Theorems 2 and 3 parallel the strange duality results for simply connected elliptic surfaces. Let

$$
\pi: Y \rightarrow \mathbb{P}^{1}
$$

be a simply connected elliptic fibration with a section and at worst irreducible nodal fibers. The dimension of the two complementary moduli spaces $\mathfrak{M}_{v}$ and $\mathfrak{M}_{w}$ will be taken large enough compared to the constant

$$
\Delta=\chi\left(Y, \mathcal{O}_{Y}\right) \cdot\left((r+s)^{2}+(r+s)+2\right)-2(r+s) .
$$

The polarization is still assumed suitable. The following was proved by combining [MOY] and $[\mathrm{BH}]$ :

Theorem. Let $v$ and $w$ be two orthogonal topological types of rank $r, s \geq 3$, such that

(i) the fiber degrees $c_{1}(v) \cdot f=c_{1}(w) \cdot f=1$,

(ii) $\operatorname{dim} \mathfrak{M}_{v}+\operatorname{dim} \mathfrak{M}_{w} \geq \Delta$.

Then,

$$
\mathrm{D}: H^{0}\left(\mathfrak{M}_{v}, \Theta_{w}\right)^{\vee} \rightarrow H^{0}\left(\mathfrak{M}_{w}, \Theta_{v}\right)
$$

is an isomorphism.

We suspect that an analogous statement can be made for all (not necessarily simply connected, with possibly reducible fibers) elliptic surfaces $Y \rightarrow C$, going beyond the scope of Theorems 2 and $2 \mathrm{~A}$.

The results for simply connected fibrations and abelian surfaces both rely on FourierMukai techniques, but the geometry is more involved in the abelian case, as already illustrated by the birationality statement of Theorem 1. Via Fourier-Mukai, instead of a rather standard analysis of tautological line bundles over Hilbert scheme of points in the 
simply connected case, one is led here to studying sections of suitable theta bundles over the schemes $\mathfrak{X}_{v}^{+}$. This requires new ideas. We prove the duality for spaces of sheaves of equal ranks and fiber degree 1, but believe these assumptions may be relaxed. The case $r=s=2$ is also left out of our theorems: while the Fourier-Mukai arguments do not cover it, we believe strange duality holds here as well.

1.4. Variation in moduli. Let $\mathcal{A}_{d}$ be the moduli space of pairs $(X, H)$, where $X$ is an abelian surface and $H$ is an ample line bundle inducing a polarization of type $(1, d)$ on $X$. For a Mukai vector $v$ with

$$
c_{1}(v)=c_{1}(H),
$$

we consider the relative moduli space of sheaves

$$
\pi: \mathfrak{M}[v]^{+} \longrightarrow \mathcal{A}_{d}
$$

whose fiber over a surface $(X, H)$ is the moduli space of $H$-semistable sheaves with Mukai vector $v$ and fixed determinant equal to $H$. Associated with two orthogonal Mukai vectors $v$ and $w$, there is a universal canonical Theta divisor

$$
\Theta_{v w}^{+}=\left\{(X, H, V, W): h^{1}(X, V \otimes W) \neq 0\right\} \subset \mathfrak{M}[v]^{+} \times{ }_{\mathcal{A}_{d}} \mathfrak{M}[w]^{+},
$$

giving rise to a line bundle which splits as a product

$$
\Theta_{v w}^{+}=\Theta_{w} \otimes \Theta_{v} \text { on } \mathfrak{M}[v]^{+} \times_{\mathcal{A}_{d}} \mathfrak{M}[w]^{+} .
$$

Pushforward via the morphisms

$$
\mathfrak{M}[v]^{+} \rightarrow \mathcal{A}_{d}, \quad \mathfrak{M}[w]^{+} \rightarrow \mathcal{A}_{d}
$$

yields two coherent sheaves of generalized theta functions over $\mathcal{A}_{d}$,

$$
\mathbb{W}=R^{0} \pi_{\star} \Theta_{w}, \quad \mathbb{V}=R^{0} \pi_{\star} \Theta_{v} .
$$

Let $\mathcal{H} \subset \mathcal{A}_{d}$ be the Humbert surface parametrizing split abelian surfaces $(X, H)$ with

$$
X=B \times F \text { and } H=L_{B} \otimes L_{F},
$$

for line bundles $L_{B}, L_{F}$ over $B$ and $F$ of degrees $d$ and 1 respectively.

Theorem 2 can be rephrased as the following generic strange duality statement:

Theorem 2R. Assume $v$ and $w$ are orthogonal Mukai vectors of equal ranks $r \geq 3$ with

(i) $c_{1}(v)=c_{1}(w)=H$;

(ii) $\langle v, v\rangle \geq 2\left(r^{2}+r-1\right),\langle w, w\rangle \geq 2\left(r^{2}+r-1\right)$. 1

\footnotetext{
${ }^{1}$ Assumption (ii) allows us to exchange $H$-stability with stability with respect to a suitable polarization, since in this case the ensuing moduli spaces agree in codimension 1 . This was proved for $K 3$ surfaces in the appendix of [MOY]. The case of abelian surfaces follows by the same argument.
} 
Then, the sheaves $\mathbb{V}$ and $\mathbb{W}$ are locally free when restricted to the Humbert surface $\mathcal{H}$, and $\Theta_{v w}^{+}$induces an isomorphism

$$
\mathrm{D}: \mathbb{W}^{\vee} \longrightarrow \mathbb{V} \text { along } \mathcal{H} \text {. }
$$

1.5. Acknowledgements. The authors acknowledge support from the NSF via grants DMS 1001604 and DMS 1001486, and thank CRM Barcelona, MPI Bonn, and the University of Bonn for their hospitality in the summer of 2012.

\section{Moduli SPACES OF SHEAVES OF FIBER DEGREE 1}

2.1. Setting. We consider a complex abelian surface $X$ which is a product of two elliptic curves,

$$
X \simeq B \times F .
$$

Letting $o_{B}, o_{F}$ denote the origins on $B$ and $F$, we write $\sigma$ and $f$ for the divisors $B \times o_{F}$ respectively $o_{B} \times F$ in the product $B \times F$. Note that

$$
\sigma^{2}=f^{2}=0, \sigma \cdot f=1
$$

2.1.1. Line bundles over $X$. For any positive integers $a$ and $b$, the line bundle $\mathcal{O}(a \sigma+b f)$ on $X$ is ample. Its higher cohomology vanishes, and we have

$$
h^{0}(X, \mathcal{O}(a \sigma+b f))=\chi(X, \mathcal{O}(a \sigma+b f))=a b .
$$

Letting

$$
\pi_{B}: X \rightarrow B, \pi_{F}: X \rightarrow F
$$

be the natural projections from $X$, we also note that for any $\ell>0$,

$$
\begin{aligned}
& h^{0}(X, \mathcal{O}(\ell \sigma))=h^{0}\left(X, \pi_{F}^{\star} \mathcal{O}\left(\ell o_{F}\right)\right)=h^{0}\left(F, \mathcal{O}\left(\ell o_{F}\right)\right)=\ell \\
& h^{0}(X, \mathcal{O}(\ell f))=h^{0}\left(X, \pi_{B}^{\star} \mathcal{O}\left(\ell o_{B}\right)\right)=h^{0}\left(B, \mathcal{O}\left(\ell o_{B}\right)\right)=\ell .
\end{aligned}
$$

Thus every section of $\mathcal{O}_{X}(\ell f)$ corresponds to a divisor of the form

$$
\bigsqcup_{i=1}^{\ell} z_{i} \times F, \text { for } z_{1}+\cdots+z_{\ell}=o_{B} .
$$

Furthermore, equations (11) and (2) imply that every section of the line bundle $\mathcal{O}_{X}(\sigma+\ell f)$ vanishes along a divisor of the form

$$
\sigma \cup \bigsqcup_{i=1}^{\ell} z_{i} \times F, \text { for } z_{1}+\cdots+z_{\ell}=o_{B} .
$$


2.1.2. Fourier-Mukai functors. Two different Fourier-Mukai transforms will be considered on $X$. First, there is the Fourier-Mukai transform with respect to the standardly normalized Poincaré bundle

$$
\mathcal{P} \rightarrow X \times \widehat{X}
$$

We identify $\widehat{X} \cong X$ using the polarization $o_{B} \times F+B \times o_{F}$. Specifically, our (perhaps non-standard) sign choice is so that $y=\left(y_{B}, y_{F}\right) \in X$ is viewed as a degree 0 line bundle $y \rightarrow X$ via the association

$$
y \mapsto \mathcal{O}_{B}\left(y_{B}-o_{B}\right) \otimes \mathcal{O}_{F}\left(y_{F}-o_{F}\right) .
$$

The Poincaré bundle is given then by

$$
\mathcal{P} \rightarrow X \times X, \quad \mathcal{P}=\mathcal{P}_{B} \otimes \mathcal{P}_{F}
$$

where

$$
\begin{gathered}
\mathcal{P}_{B} \rightarrow B \times B, \quad \mathcal{P}_{B}=\mathcal{O}_{B \times B}\left(\Delta_{B}\right) \otimes p^{\star} \mathcal{O}_{B}\left(-o_{B}\right) \otimes q^{\star} \mathcal{O}_{B}\left(-o_{B}\right), \text { and } \\
\mathcal{P}_{F} \rightarrow F \times F, \quad \mathcal{P}_{F}=\mathcal{O}_{F \times F}\left(\Delta_{F}\right) \otimes p^{\star} \mathcal{O}_{F}\left(-o_{F}\right) \otimes q^{\star} \mathcal{O}_{F}\left(-o_{F}\right) .
\end{gathered}
$$

The Fourier-Mukai transform with kernel $\mathcal{P}$ is denoted

$$
\mathbf{R S}: \mathbf{D}(X) \rightarrow \mathbf{D}(X) .
$$

A second Fourier-Mukai transform is defined by considering the relative Picard variety of $\pi_{B}: X \rightarrow B$. We identify $F \cong \widehat{F}$ so that

$$
y_{F} \in F \mapsto \mathcal{O}_{F}\left(y_{F}-o_{F}\right),
$$

and we let

$$
\mathbf{R} \mathcal{S}^{\dagger}: \mathbf{D}(X) \rightarrow \mathbf{D}(X)
$$

be the Fourier-Mukai transform whose kernel is the pullback of the Poincaré sheaf

$$
\mathcal{P}_{F} \rightarrow F \times F
$$

to the product $X \times_{B} X \cong F \times F \times B$. Both Fourier-Mukai transforms are known to be equivalences of derived categories.

Several properties of the Fourier-Mukai will be used below. First, for integers $a, b$ we have

$$
\operatorname{det} \mathbf{R} \mathcal{S}(\mathcal{O}(a \sigma+b f))=\mathcal{O}(-b \sigma-a f)
$$

and

$$
\operatorname{det} \mathbf{R} \mathcal{S}^{\dagger}(\mathcal{O}(a \sigma+b f))=\mathcal{O}(-\sigma+a b f) .
$$

Next, we have the following standard result, which will in fact be proved in greater generality in Lemma 1A of Section 3 . 
Lemma 1. For $x=\left(x_{B}, x_{F}\right) \in X, y=\left(y_{B}, y_{F}\right) \in \widehat{X}$, we have

$$
\begin{gathered}
\mathbf{R} \mathcal{S}^{\dagger}\left(t_{x}^{\star} E\right)=t_{x_{B}}^{\star} \mathbf{R} \mathcal{S}^{\dagger}(E) \otimes x_{F}^{\vee}, \\
\mathbf{R} \mathcal{S}^{\dagger}(E \otimes y)=t_{y_{F}}^{\star} \mathbf{R} \mathcal{S}^{\dagger}(E) \otimes y_{B} .
\end{gathered}
$$

2.2. Moduli spaces of sheaves. We consider sheaves over $X$ of Mukai vector $v$ such that

$$
\operatorname{rank} v=r, \quad \chi(v)=\chi, c_{1}(v) \cdot f=1 .
$$

We set

$$
d_{v}=\frac{1}{2}\langle v, v\rangle=\frac{c_{1}(v)^{2}}{2}-r \chi
$$

which is half the dimension of the moduli space $\mathfrak{M}_{v}^{+}$below. Recall from the introduction that the polarization $H$ is suitable i.e.,

$$
H=\sigma+N f, \text { for } N \gg 0
$$

We are concerned with three moduli spaces of sheaves:

(i) The moduli space $K_{v}$ of $H$-semistable sheaves $V$ on $X$ with

$$
\operatorname{det} V=\mathcal{O}(\sigma+m f), \operatorname{det} \mathbf{R} \mathcal{S}(V)=\mathcal{O}(-m \sigma-f) .
$$

Thus the determinants of the sheaves and of the Fourier-Mukai transforms of sheaves in $K_{v}$ are fixed. Here, we wrote

$$
c_{1}(v)=\sigma+m f
$$

where

$$
m=d_{v}+r \chi
$$

(ii) The moduli space $\mathfrak{M}_{v}^{+}$of $H$-semistable sheaves $V$ on $X$ with

$$
\operatorname{det} V=\mathcal{O}(\sigma+m f) \text {. }
$$

The two spaces (i) and (ii) are related via the degree $d_{v}^{4}$ étale morphism

$$
\Phi_{v}^{+}: K_{v} \times X \rightarrow \mathfrak{M}_{v}^{+}, \quad \Phi_{v}^{+}(V, x)=t_{r x}^{\star} V \otimes t_{x}^{\star} \operatorname{det} V^{-1} \otimes \operatorname{det} V .
$$

In explicit form, we write equivalently

$$
\Phi_{v}^{+}(V, x)=t_{r x}^{\star} V \otimes\left(x_{F} \otimes x_{B}^{m}\right)
$$

(iii) Finally, there is a moduli space $\mathfrak{M}_{v}^{-}$of semistable sheaves whose Fourier-Mukai transform has fixed determinant

$$
\operatorname{det} \mathbf{R} \mathcal{S}(V)=\mathcal{O}(-f-m \sigma) .
$$


In this case, we shall make use of the étale morphism

$$
\Phi_{v}^{-}: K_{v} \otimes \widehat{X} \rightarrow \mathfrak{M}_{v}^{-}, \quad \Phi_{v}^{-}(V, y)=t_{\left(y_{B}, m y_{F}\right)}^{\star} V \otimes y^{\chi} .
$$

Note that we have indeed

$$
\begin{aligned}
\operatorname{det} \mathbf{R} \mathcal{S} \Phi_{v}^{-}(V, y) & =\operatorname{det} \mathbf{R} \mathcal{S}\left(t_{\left(y_{B}, m y_{F}\right)}^{\star} V \otimes y^{\chi}\right)=t_{\chi y}^{\star} \operatorname{det} \mathbf{R} \mathcal{S}\left(t_{\left(y_{B}, m y_{F}\right)}^{\star} V\right) \\
& =t_{\chi y}^{\star} \operatorname{det}\left(\mathbf{R} \mathcal{S}(V) \otimes\left(y_{B}, m y_{F}\right)^{-1}\right)=t_{\chi y}^{\star} \mathcal{O}(-f-m \sigma) \otimes\left(y_{B}, m y_{F}\right)^{-\chi} \\
& =\mathcal{O}(-f-m \sigma) .
\end{aligned}
$$

For further details regarding the morphisms $\Phi_{v}^{+}, \Phi_{v}^{-}$, we refer the reader to Sections 4 and 5 of $\mathrm{MO} 2$.

2.3. Birationality of $K_{v}$ with the generalized Kummer variety $K^{\left[d_{v}\right]}$. We now establish in two different ways an explicit birational map

$$
\Psi_{r}: K^{\left[d_{v}\right]} \rightarrow K_{v}
$$

where $K^{\left[d_{v}\right]}$ is the generalized Kummer variety of dimension $2 d_{v}-2$,

$$
K^{\left[d_{v}\right]}=\left\{Z \in X^{\left[d_{v}\right]}: a(Z)=0\right\} .
$$

As usual, $a: X^{\left[d_{v}\right]} \rightarrow X$ denotes the addition map on the Hilbert scheme.

2.3.1. O'Grady's construction. We first obtain the map $\Psi_{r}$ by induction on the rank $r$ of the sheaves, following O'Grady's work $\mathrm{OG}$. for elliptically fibered $K 3$ surfaces. To start the induction, we let $Z \subset X$ be a zero dimensional subscheme of length

$$
\ell=\ell(Z)=d_{v},
$$

such that $a(Z)=0$, and further satisfying the following conditions:

(i) $Z$ does not contain two points in the same fiber of $\pi_{B}$,

(ii) $Z$ does not intersect the section $\sigma$.

Such a $Z$ is a generic point in the generalized Kummer variety $K^{\left[d_{v}\right]}$. We let

$$
m_{1}=\chi+d_{v}
$$

which the correct number of fibers needed when $r=1$, and set

$$
V_{1}=I_{Z} \otimes \mathcal{O}\left(\sigma+m_{1} f\right) .
$$

Note that $\chi\left(V_{1}\right)=\chi$, and that $V_{1}$ thus constructed belongs to $K_{v}$. The requirement

$$
\operatorname{det} \mathbf{R} \mathcal{S}\left(V_{1}\right)=\mathcal{O}\left(-m_{1} \sigma-f\right),
$$

is a consequence of the fact that $a(Z)=0$. 
We claim that for $Z$ as above we have

$$
h^{1}\left(V_{1}(-\chi f)\right)=h^{1}\left(I_{Z} \otimes \mathcal{O}(\sigma+\ell f)\right)=h^{0}\left(I_{Z}(\sigma+\ell f)\right)=1 .
$$

Indeed, as explained in Section 2.1, every section of $\mathcal{O}_{X}(\sigma+\ell f)$ vanishes along a divisor of the form

$$
\sigma+\pi_{B}^{\star}\left(z_{1}+\ldots z_{\ell}\right)
$$

where $z_{1}, \ldots, z_{\ell}$ are points of $B$ such that $z_{1}+\ldots+z_{\ell}=o_{B}$. Conditions (i) and (ii) ensure that there is a unique such divisor passing through $Z: z_{1}, \ldots, z_{\ell}$ are the $B$-coordinates of the distinct points of $Z$.

We inductively construct extensions

$$
0 \rightarrow \mathcal{O}(\chi f) \rightarrow V_{r+1} \rightarrow V_{r} \rightarrow 0
$$

with stable middle term. The sheaves obtained will satisfy

$$
\chi\left(V_{r}(-\chi f)\right)=0 .
$$

In order to get (5), we show

$$
\operatorname{ext}^{1}\left(V_{r}, \mathcal{O}(\chi f)\right)=h^{1}\left(V_{r}(-\chi f)\right)=h^{0}\left(V_{r}(-\chi f)\right)=1 .
$$

For $r=1$, the ext-dimension is 1 by (44). Assuming the statement for $r$, we get the unique nontrivial extension, and argue for stability of the middle term $V_{r+1}$. Indeed, Proposition I.4.7 of $[\mathrm{OG}$ ] gives stability of the middle term and also asserts the restrictions of (5) to any fibers of $\pi$ do not split, if a suitable vanishing hypothesis holds. Precisely, we require that for all fibers $f$, we have

$$
\operatorname{Hom}\left(\left.V_{r}\right|_{\mathrm{f}}, \mathcal{O}_{\mathrm{f}}\right)=0
$$

The fiber restrictions are calculated as in Lemma 1 in [MOY] as follows:

(i) the restriction of the defining exact sequence to any fiber $f_{\eta}$ yields

$$
\left.\left.0 \rightarrow \mathcal{O}_{f_{\eta}} \rightarrow V_{r+1}\right|_{f_{\eta}} \rightarrow V_{r}\right|_{f_{\eta}} \rightarrow 0 .
$$

Hence inductively, for fibers avoiding $Z$, we have that

$$
\left.V_{r}\right|_{f_{\eta}} \cong \mathrm{E}_{r, o},
$$

the Atiyah bundle of rank $r$ and determinant $\mathcal{O}_{f_{\eta}}(o)$.

(ii) for fibers $f_{z}$ containing points $z \in Z$ we have

$$
\left.V_{r}\right|_{f_{z}}=\mathrm{E}_{r-1, z} \oplus \mathcal{O}_{f_{z}}(o-z) .
$$


As a consequence of equations (77) and (86), the vanishing (66) stated above is satisfied.

To complete the argument, we establish now that there exists a unique extension (5) for $r+1$ i.e., we show $h^{1}\left(V_{r+1}(-\chi f)\right)=1$. First, stability of $V_{r}$ and $V_{r+1}$ ensures that

$$
H^{2}\left(V_{r}(-\chi f)\right)=H^{2}\left(V_{r+1}(-\chi f)\right)=0,
$$

and the cohomology exact sequence of the unique extension for $r$ is

$$
\begin{aligned}
0 \rightarrow & H^{0}(\mathcal{O}) \rightarrow H^{0}\left(V_{r+1}(-\chi f)\right) \rightarrow H^{0}\left(V_{r}(-\chi f)\right) \rightarrow H^{1}(\mathcal{O}) \\
& \rightarrow H^{1}\left(V_{r+1}(-\chi f)\right) \rightarrow H^{1}\left(V_{r}(-\chi f)\right) \rightarrow H^{2}(\mathcal{O}) \rightarrow 0 .
\end{aligned}
$$

By dimension counting, the last map has to be a bijection, hence $H^{1}(\mathcal{O}) \rightarrow H^{1}\left(V_{r+1}(-\chi f)\right)$ is surjective. We claim this map cannot be a bijection. Indeed, if $H^{1}(\mathcal{O}) \rightarrow H^{1}\left(V_{r+1}(-\chi f)\right)$ were bijective, we would have that

$$
H^{0}\left(V_{r}(-\chi f)\right) \rightarrow H^{1}(\mathcal{O})
$$

is the zero map. But this map is simply multiplication by the extension class which is nontrivial. Since $h^{0}\left(V_{r+1}(-\chi f)\right) \geq 1$ from the same sequence, and $H^{0}\left(V_{r+1}(-\chi f)\right)=$ $H^{1}\left(V_{r+1}(-\chi f)\right)$, we conclude both vector spaces are of dimension 1 , as needed.

Finally, the argument clearly shows that the map $\Psi_{r}$ is injective. Therefore, we obtain a birational map

$$
\Psi_{r}: K^{\left[d_{v}\right]} \rightarrow K_{v},
$$

by the equality of dimensions for the two irreducible spaces.

2.3.2. Fourier-Mukai construction. We next point out that $\Psi_{r}$ can also be viewed as a fiberwise Fourier-Mukai transform. This is similar to the case of $K 3$ surfaces analyzed in [MOY]. We show

Proposition 1. For subschemes $Z$ with $a(Z)=0$, satisfying (i) and (ii), we have

$$
\mathbf{R} \mathcal{S}^{\dagger}\left(V_{r}^{\vee}\right)=I_{\widetilde{Z}}(r \sigma-\chi f)[-1]
$$

and

$$
\mathbf{R} \mathcal{S}^{\dagger}\left(V_{r}\right)=I_{Z}^{\vee}(-r \sigma+\chi f)
$$

where $\widetilde{Z}$ is the reflection of $Z$ along the zero section $\sigma$.

Proof. We prove the first equality. By the calculation of the restrictions of $V_{r}$ to the fibers in equations (77) and (8), it follows that $V_{r}^{\vee}$ contains no subbundles of positive degree over each fiber. Therefore, $\mathbf{R} \mathcal{S}^{\dagger}\left(V_{r}^{\vee}\right)[1]$ is torsion free, by Proposition 3.7 of [BH]. The agreement of $\mathbf{R} \mathcal{S}^{\dagger}\left(V_{r}^{\vee}\right)[1]$ and $I_{\widetilde{Z}}(r \sigma-\chi f)$ holds fiberwise. This can be seen using (7) 
and (8), just as in Proposition 1 in [MOY]. The proof is completed by proving equality of determinants. In turn, this follows inductively from the exact sequence (5):

$$
\operatorname{det} \mathbf{R} \mathcal{S}^{\dagger}\left(V_{r+1}^{\vee}\right)=\operatorname{det} \mathbf{R} \mathcal{S}^{\dagger}\left(V_{r}^{\vee}\right) \otimes \operatorname{det} \mathbf{R} \mathcal{S}^{\dagger}(\mathcal{O}(-\chi f))=\operatorname{det} \mathbf{R} \mathcal{S}^{\dagger}\left(V_{r}^{\vee}\right) \otimes \mathcal{O}(-\sigma) .
$$

The base case $r=1$ is immediate, as

$$
\begin{gathered}
\operatorname{det} \mathbf{R} \mathcal{S}^{\dagger}\left(I_{Z}^{\vee}\left(-\sigma-m_{1} f\right)\right)=\operatorname{det} \mathbf{R} \mathcal{S}^{\dagger}\left(\mathcal{O}\left(-\sigma-m_{1} f\right)\right) \otimes_{z \in Z} \operatorname{det}\left(\mathbf{R} \mathcal{S}^{\dagger}\left(\mathcal{O}_{z}^{\vee}\right)\right)^{\vee} \\
=\mathcal{O}\left(-\sigma+m_{1} f\right) \otimes_{z \in Z} \operatorname{det}\left(\mathbf{R} \mathcal{S}^{\dagger}\left(\mathcal{O}_{z}[-2]\right)\right)^{\vee}=\mathcal{O}\left(-\sigma+m_{1} f\right) \otimes_{z \in Z} \mathcal{O}\left(-f_{z}\right) \\
=\mathcal{O}\left(-\sigma+m_{1} f\right) \otimes \mathcal{O}(-\ell f)=\mathcal{O}(-\sigma+\chi f)
\end{gathered}
$$

using that $a(Z)=0$. Here $f_{z}$ denotes the fiber through $z \in Z$. A similar calculation can be carried out for the Fourier-Mukai with kernel $\mathcal{P}^{\vee}$.

The second statement follows then by Grothendieck duality. We refer the reader to Proposition 2 in [MOY] for an identical computation.

Proposition 1 gives an explicit description of O'Grady's construction in terms of the Fourier-Mukai transform, at least away from the divisors (i) and (ii). Explicitly, under the correspondence

$$
K^{\left[d_{v}\right]} \ni Z \mapsto I_{\tilde{Z}}(r \sigma-\chi f)[-1],
$$

the birational map $\Psi_{r}: K^{\left[d_{v}\right]} \rightarrow-K_{v}$ is given by the inverse of $\mathbf{R} \mathcal{S}^{\dagger}$, followed by dualizing

$$
\Psi_{r} \cong \mathbf{D}_{X} \circ\left(\mathbf{R} \mathcal{S}^{\dagger}\right)^{-1}
$$

However, viewed as a Fourier-Mukai transform, $\Psi_{r}$ extends to an isomorphism in codimension 1 , whenever $r \geq 3$. This is established in Sections 3 and 5 of $[\mathrm{BH}]$ on general grounds, but can also be argued directly.

Indeed, the inverse of $\mathbf{R} \mathcal{S}^{\dagger}$ is, up to shifts, the Fourier-Mukai transform with kernel the dual $\pi_{F \times F}^{\star} \mathcal{P}_{F} \vee$ of the fiberwise Poincaré line bundle on $X \times_{B} X \simeq F \times F \times B$. We claim that as long as $r \geq 3$ and $Z$ contains no more than two points in the same fiber of

$$
\pi_{B}: X \rightarrow B
$$

the Fourier-Mukai image of the sheaf $I_{Z}(r \sigma-\chi f)$ is a vector bundle. Stability is automatic, as the restrictions to all elliptic fibers which do not pass through $Z$ are isomorphic to the Atiyah bundle of rank $r$ and degree 1, therefore the restriction to the generic fiber is stable. The locus of $Z \in K^{\left[d_{v}\right]}$ with at least 3 points in the same elliptic fiber has codimension 2 . 
To see the claim above, note that the restriction of the ideal sheaf of a point to the elliptic fiber $f$ through that point is

$$
\left.I_{p}\right|_{\mathrm{f}}=\mathcal{O}_{\mathrm{f}}(-p)+\mathcal{O}_{p},
$$

and similarly

$$
\left.I_{p, p^{\prime}}\right|_{\mathrm{f}}=\mathcal{O}_{\mathrm{f}}\left(-p-p^{\prime}\right)+\mathcal{O}_{p}+\mathcal{O}_{p^{\prime}},
$$

for (possibly coincident) points $p, p^{\prime}$ in the fiber $\mathrm{f}$. Thus, the restriction of $I_{Z}(r \sigma-\chi f)$ to fibers $f$ can take the form

$$
\mathcal{O}_{\mathrm{f}}\left(r o_{F}\right), \quad \mathcal{O}_{\mathrm{f}}\left(r o_{F}\right) \otimes\left(\mathcal{O}_{\mathrm{f}}(-p)+\mathcal{O}_{p}\right) \text { or } \mathcal{O}_{\mathrm{f}}\left(r o_{F}\right) \otimes\left(\mathcal{O}_{\mathrm{f}}\left(-p-p^{\prime}\right)+\mathcal{O}_{p}+\mathcal{O}_{p^{\prime}}\right)
$$

which are all $I T_{0}$ with respect to $\mathcal{P}_{F}^{\vee}$, for $r \geq 3$. Thus the Fourier-Mukai transform $\left(\mathbf{R} \mathcal{S}^{\dagger}\right)^{-1}$ of $I_{Z}(r \sigma-\chi f)[-1]$ is a vector bundle by cohomology and base-change.

When $r=2, \Psi_{2}$ is defined away from the divisor of subschemes $Z \in K^{\left[d_{v}\right]}$ with at least two points in the same elliptic fiber. As $K^{\left[d_{v}\right]}$ and $K_{v}$ are irreducible holomorphic symplectic, $\Psi_{2}$ extends anyway to a birational map which is regular outside of codimension 2, but this extension is no longer identical to the Fourier-Mukai transform. In fact, semistable reduction is necessary to construct the extension, as in Section I.4 of OG. We will not pursue it in this paper.

2.4. The moduli space $\mathfrak{M}_{v}^{+}$via Fourier-Mukai. We investigate how sheaves of fixed determinant change under Fourier-Mukai. Recall the morphism (3), and let

$$
V=\Phi_{v}^{+}(E, x),
$$

for a pair $(E, x) \in K_{v} \times X$. Using Lemma 1 and Proposition 1, we calculate

$$
\begin{aligned}
\mathbf{R} \mathcal{S}^{\dagger}(V) & =\mathbf{R} \mathcal{S}^{\dagger}\left(t_{r x}^{\star} E \otimes\left(x_{B}^{m} \otimes x_{F}\right)\right)=t_{x_{F}}^{\star} \mathbf{R} \mathcal{S}^{\dagger}\left(t_{r x}^{\star} E\right) \otimes x_{B}^{m} \\
& =t_{x_{F}}^{\star}\left(t_{r x_{B}}^{\star} \mathbf{R} \mathcal{S}^{\dagger}(E) \otimes x_{F}^{-r}\right) \otimes x_{B}^{m} \\
& =t_{x_{F}+r x_{B}}^{\star}\left(I_{Z}^{\vee}(-r \sigma+\chi f)\right) \otimes\left(x_{F}^{-r} \otimes x_{B}^{m}\right) \\
& =I_{Z^{+}}^{\vee}(-r \sigma+\chi f) \otimes x_{B}^{d_{v}},
\end{aligned}
$$

where

$$
Z^{+}=t_{x_{F}+r x_{B}}^{\star} Z, \text { so that } a_{B}\left(Z^{+}\right)=-d_{v} r x_{B} .
$$

In a similar fashion, we prove that

$$
\mathbf{R} \mathcal{S}^{\dagger}\left(V^{\vee}\right)=I_{Z_{+}}(r \sigma-\chi f)[-1] \otimes x_{B}^{-d_{v}},
$$

where now

$$
Z_{+}=t_{-x_{F}+r x_{B}}^{\star} \widetilde{Z}=\widetilde{Z^{+}}
$$


From the first equation, we obtain the rational map

$$
\mathbf{R} \mathcal{S}^{\dagger}: K_{v} \times X \rightarrow \mathfrak{X}_{v}^{+}
$$

where

$$
\mathfrak{X}_{v}^{+}=\left\{\left(Z^{+}, z_{B}\right): a_{B}\left(Z^{+}\right)=r z_{B}\right\} \subset X^{\left[d_{v}\right]} \times B
$$

via the assignment

$$
(E, x) \mapsto\left(Z^{+},-d_{v} x_{B}\right) .
$$

This map has degree $d_{v}^{4}$ and descends to $\mathfrak{M}_{v}^{+}$. Since $\Phi_{v}^{+}$is also of degree $d_{v}^{4}$ and $\mathfrak{X}_{v}^{+}$is irreducible of the same dimension as $\mathfrak{M}_{v}^{+}$, we obtain a birational isomorphism

$$
\mathfrak{M}_{v}^{+}-\rightarrow \mathfrak{X}_{v}^{+}
$$

in such a fashion that

$$
\mathbf{R} \mathcal{S}^{\dagger}(V)=I_{Z^{+}}^{\vee}(-r \sigma+\chi f) \otimes z_{B}^{-1}
$$

and

$$
\mathbf{R} \mathcal{S}^{\dagger}\left(V^{\vee}\right)=I_{\widetilde{Z^{+}}}(r \sigma-\chi f) \otimes z_{B}[-1] .
$$

The discussion of the previous subsection shows the birational isomorphism is given (explicitly as a Fourier-Mukai transform) away from codimension 2.

2.5. The moduli space $\mathfrak{M}_{v}^{-}$via Fourier-Mukai. A similar argument applies to the moduli space $\mathfrak{M}_{v}^{-}$of sheaves with fixed determinant of their Fourier-Mukai transform

$$
\operatorname{det} \mathbf{R} \mathcal{S}(V)=\mathcal{O}(-f-m \sigma) .
$$

In this case, we have a morphism

$$
\Phi_{v}^{-}: K_{v} \otimes \widehat{X} \rightarrow \mathfrak{M}_{v}^{-}
$$

given by

$$
\Phi_{v}^{-}(E, y)=t_{\left(y_{B}, m y_{F}\right)}^{\star} E \otimes y^{\chi} .
$$

We calculate

$$
\begin{aligned}
\mathbf{R} \mathcal{S}^{\dagger}\left(\Phi_{v}^{-}(E, y)\right) & =\mathbf{R} \mathcal{S}^{\dagger}\left(t_{\left(y_{B}, m y_{F}\right)}^{\star} E \otimes y^{\chi}\right)=t_{\chi y_{F}}^{\star} \mathbf{R S}^{\dagger}\left(t_{\left(y_{B}, m y_{F}\right)}^{\star} E\right) \otimes y_{B}^{\chi} \\
& =t_{\chi y_{F}}^{\star}\left(t_{y_{B}}^{\star} \mathbf{R} \mathcal{S}^{\dagger}(E) \otimes y_{F}^{-m}\right) \otimes y_{B}^{\chi} \\
& =t_{y_{B}+\chi y_{F}}^{\star}\left(I_{Z}^{\vee}(-r \sigma+\chi f)\right) \otimes\left(y_{F}^{-m} \otimes y_{B}^{\chi}\right) \\
& =I_{Z^{-}}^{\vee}(-r \sigma+\chi f) \otimes y_{F}^{-d_{v}}
\end{aligned}
$$

where $Z^{-}=t_{y_{B}+\chi y_{F}}^{\star} Z$, so that the addition in the fibers is $a_{F}\left(Z^{-}\right)=-\chi d_{v} y_{F}$. We therefore obtain the birational isomorphism

$$
\mathfrak{M}_{v}^{-}-\rightarrow \mathfrak{X}_{v}^{-}, \quad \Phi_{v}^{-}(E, y) \mapsto\left(Z^{-},-d_{v} y_{F}\right)
$$


where

$$
\mathfrak{X}_{v}^{-}=\left\{\left(Z^{-}, z_{F}\right): a_{F}\left(Z^{-}\right)=\chi z_{F}\right\} \hookrightarrow X^{\left[d_{v}\right]} \times F .
$$

For further use, we record the following identities

$$
\mathbf{R} \mathcal{S}^{\dagger}(V)=I_{Z^{-}}^{\vee}(-r \sigma+\chi f) \otimes z_{F}, \mathbf{R} \mathcal{S}^{\dagger}\left(V^{\vee}\right)=I_{\widetilde{Z^{-}}}(r \sigma-\chi f) \otimes z_{F}[-1]
$$

\section{RANK-COPRIME ARBITRARY FIBER DEGREE}

Theorem 1 was proved in the previous Section for fiber degree 1 in two ways: via an explicit analysis of O'Grady's description of the moduli space, and via Fourier-Mukai methods. In this section, we use Fourier-Mukai to prove Theorem 1 for arbitrary fiber degree. The argument builds on results of Bridgeland $[\mathrm{B}]$. At the end of the section, we briefly consider the case of a surface $X=C \times F$ with $F$ elliptic, but $C$ of higher genus.

3.1. Fourier-Mukai transforms in the general coprime setting. We write

$$
d=c_{1}(v) \cdot f
$$

for the fiber degree, which we assume to be coprime to the rank $r$. Thus $c_{1}(v)=d \sigma+m f$. Pick integers $a$ and $b$ such that

$$
a d+b r=1,
$$

with $0<a<r$. The following lemma gives the kernel of the Fourier-Mukai transform we will use:

Lemma 2. There exists a vector bundle

$$
\mathcal{U} \rightarrow F \times F
$$

with the following properties:

(i) the restriction of $\mathcal{U}$ to $F \times\{y\}$ is stable of rank a and degree $b$;

(ii) the restriction of $\mathcal{U}$ to $\{x\} \times F$ is stable of rank a and degree $r$.

Furthermore,

$$
c_{1}(\mathcal{U})=b\left[o_{F} \times F\right]+r\left[F \times o_{F}\right]+c_{1}\left(\mathcal{P}_{F}\right) .
$$

Proof. This result is known, see for instance [B], and can be explained in several ways. We consider the moduli space $M_{F}(a, b)$ of bundles of rank $a$ and degree $b$ over $F$. By the classic result of Atiyah [A], we have

$$
M_{F}(a, b) \cong F .
$$

We let

$$
\mathcal{U} \rightarrow F \times F
$$


denote the universal bundle. Therefore, for all $y \in F$, we have that $\left.\mathcal{U}\right|_{E \times y}$ has rank $a$ and degree $b$; in fact the determinant equals $\mathcal{O}_{F}\left((b-1) \cdot o_{F}+y\right)$. The bundle $\mathcal{U}$ is not unique and we can normalize it in several ways. Indeed, for any matrix

$$
A=\left[\begin{array}{ll}
\lambda & a \\
\mu & b
\end{array}\right] \in S L_{2}(\mathbb{Z})
$$

we may assume that $\left.\mathcal{U}\right|_{x \times F}$ has type $(a, \lambda)$. In fact, we can regard the first factor $F$ as the moduli space of bundles of rank $a$ and degree $\lambda$ over the second factor $F$, cf. $[\mathrm{B}$. We may pick the pair $\lambda=r$ and $\mu=-d$. What we showed above allows us to conclude $c_{1}(\mathcal{U})=b\left[o_{F} \times F\right]+r\left[F \times o_{F}\right]+c_{1}\left(\mathcal{P}_{F}\right)$. Replacing $\mathcal{U}$ by a suitable twist, we may in fact achieve

$$
\operatorname{det} \mathcal{U}=\mathcal{O}\left(b\left[o_{F} \times F\right]+r\left[F \times o_{F}\right]\right) \otimes \mathcal{P}_{F} .
$$

The lemma is proved.

Lemma 3. The sheaf $\mathcal{U} \rightarrow F \times F$ is semihomogeneous. More precisely,

$$
t_{(x, y)}^{\star} \mathcal{U}=\mathcal{U} \otimes \pi_{1}^{\star}\left(x^{-\frac{b}{a}} y^{\frac{1}{a}}\right) \otimes \pi_{2}^{\star}\left(x^{\frac{1}{a}} y^{-\frac{r}{a}}\right) .
$$

In particular,

$$
\operatorname{ch} \mathcal{U}=a \exp \left(\frac{c_{1}(\mathcal{U})}{a}\right) \Longrightarrow \chi(\mathcal{U})=-d
$$

Proof. By symmetry it suffices to argue that

$$
t_{(x, 0)}^{\star} \mathcal{U}=\mathcal{U} \otimes \pi_{1}^{\star} x^{-\frac{b}{a}} \otimes \pi_{2}^{\star} x^{\frac{1}{a}} .
$$

We note that the choice of roots for the line bundles on the right hand side is not relevant. The restrictions of both sides to $F \times\{y\}$ agree: $\mathcal{U}_{y}=\left.\mathcal{U}\right|_{F \times\{y\}}$ is stable on $F$, and thus semihomogeneous, satisfying

$$
t_{x}^{\star} \mathcal{U}_{y}=\mathcal{U}_{y} \otimes x^{-\frac{b}{a}}
$$

We check agreement over $o_{F} \times F$. This is the statement that

$$
\left.\mathcal{U}\right|_{x \times F}=\left.\mathcal{U}\right|_{o \times F} \otimes x^{\frac{1}{a}}
$$

which holds by comparing ranks and determinants. Finally, agreement over $F \times F$ follows from the generalized see-saw Lemma 2.5 of Ramanan $[\mathrm{R}$.

The second part of the lemma concerning the numerical invariants of $\mathcal{U}$ follows from general facts about semihomogeneous bundles [M].

Remark 1. A family of semihomogeneous bundles with fixed numerical invariants were constructed in arbitrary dimension in [0], and played a role in the decomposition of the Verlinde bundles. The dimension 1 case specializes to the bundle $\mathcal{U}$ considered here. 
Letting $\pi_{F \times F}: F \times F \times B \rightarrow F \times F$ be the projection, we consider the Fourier-Mukai transform

$$
\mathbf{R} \mathcal{S}^{\dagger}: \mathbf{D}(X) \rightarrow \mathbf{D}(X)
$$

with kernel

$$
\pi_{F \times F}^{\star} \mathcal{U} \rightarrow F \times F \times B \cong X \times{ }_{B} X .
$$

It follows from [B] that the kernel $\mathcal{U}$ is strongly simple over each factor, hence the Fourier-Mukai transform $\mathbf{R} \mathcal{S}^{\dagger}$ is an equivalence, with inverse having kernel $\pi_{F \times F}^{\star} \mathcal{U}^{\vee}[1]$.

\section{Lemma 1A.}

$$
\begin{aligned}
\mathbf{R} \mathcal{S}^{\dagger}(E \otimes y) & =t_{a y_{F}}^{\star} \mathbf{R} \mathcal{S}^{\dagger}(E) \otimes\left(y_{B} \otimes y_{F}^{r}\right), \\
\mathbf{R} \mathcal{S}^{\dagger}\left(t_{x}^{\star} E\right) & =t_{x_{B}+b x_{F}}^{\star} \mathbf{R} \mathcal{S}^{\dagger}(E) \otimes x_{F}^{-d} .
\end{aligned}
$$

Proof. For both formulas, it is enough to consider the case of split sheaves

$$
E=G \otimes H,
$$

where $G, H$ are sheaves over $B$ and $F$ 2. Clearly,

$$
\mathbf{R} \mathcal{S}^{\dagger}(E)=G \otimes \mathbf{R} \pi_{2 \star}\left(\pi_{1}^{\star} H \otimes \mathcal{U}\right),
$$

while

$$
\mathbf{R} \mathcal{S}^{\dagger}(E \otimes y)=\left(G \otimes y_{B}\right) \otimes \mathbf{R} \pi_{2 \star}\left(\pi_{1}^{\star}\left(H \otimes y_{F}\right) \otimes \mathcal{U}\right) .
$$

For the first formula, it suffices now to explain that

$$
\mathbf{R} \pi_{2 \star}\left(\pi_{1}^{\star}\left(H \otimes y_{F}\right) \otimes \mathcal{U}\right)=t_{a y_{F}}^{\star} \mathbf{R} \pi_{2 \star}\left(\pi_{1}^{\star} H \otimes \mathcal{U}\right) \otimes y_{F}^{r} .
$$

This is however clear since from (9),

$$
\mathcal{U} \otimes \pi_{1}^{\star} y_{F}=t_{\left(0, a y_{F}\right)}^{\star} \mathcal{U} \otimes \pi_{2}^{\star} y_{F}^{r} .
$$

We now explain the second formula by assuming as before that $E$ splits. We calculate

$$
\mathbf{R} \mathcal{S}^{\dagger}\left(t_{x}^{\star} E\right)=\mathbf{R} \mathcal{S}^{\dagger}\left(t_{x_{B}}^{\star} G \otimes t_{x_{F}}^{\star} H\right)=t_{x_{B}}^{\star} G \otimes \mathbf{R} \pi_{2 \star}\left(\pi_{1}^{\star} t_{x_{F}}^{\star} H \otimes \mathcal{U}\right) .
$$

It remains to argue that

$$
\mathbf{R} \pi_{2 \star}\left(\pi_{1}^{\star} t_{x_{F}}^{\star} H \otimes \mathcal{U}\right)=t_{b x_{F}}^{\star} \mathbf{R} \pi_{2 \star}\left(\pi_{1}^{\star} H \otimes \mathcal{U}\right) \otimes x_{F}^{-d} .
$$

Indeed, (9) gives

$$
t_{\left(x_{F}, 0\right)}^{\star} \mathcal{U}=\mathcal{U} \otimes \pi_{1}^{\star} x_{F}^{-\frac{b}{a}} \otimes \pi_{2}^{\star} x_{F}^{\frac{1}{a}},
$$

\footnotetext{
${ }^{2}$ Indeed, both left and right hand sides of the equalities claimed by the Lemma are Fourier-Mukai equivalences (note that translations and tensorization are particular examples of Fourier-Mukai transforms, and composition of Fourier-Mukai transforms is one as well). Taking inverses, it suffices to prove that if a Fourier-Mukai equivalence is the identity over split sheaves of the type $G \otimes H$, then it is always the identity. But this is clear, as one proves that the kernel of such a transform is the structure sheaf of the diagonal.
} 
so we calculate

$$
\begin{aligned}
\mathbf{R} \pi_{2 \star}\left(\pi_{1}^{\star} t_{x_{F}}^{\star} H \otimes \mathcal{U}\right) & =\mathbf{R} \pi_{2 \star}\left(t_{\left(x_{F}, 0\right)}^{\star}\left(\pi_{1}^{\star} H \otimes \mathcal{U}\right) \otimes \pi_{1}^{\star} x_{F}^{\frac{b}{a}} \otimes \pi_{2}^{\star} x_{F}^{-\frac{1}{a}}\right) \\
& =\mathbf{R} \pi_{2 \star}\left(t_{\left(x_{F}, 0\right)}^{\star}\left(\pi_{1}^{\star}\left(H \otimes x_{F}^{\frac{b}{a}}\right) \otimes \mathcal{U}\right)\right) \otimes x_{F}^{-\frac{1}{a}} \\
& =\mathbf{R} \pi_{2 \star}\left(\pi_{1}^{\star}\left(H \otimes x_{F}^{\frac{b}{a}}\right) \otimes \mathcal{U}\right) \otimes x_{F}^{-\frac{1}{a}} \\
& =t_{b x_{F}}^{\star} \mathbf{R} \pi_{2 \star}\left(\pi_{1}^{\star}(H) \otimes \mathcal{U}\right) \otimes x_{F}^{\frac{b r}{a}} x_{F}^{\frac{-1}{a}} \text { (using (10) ) } \\
& =t_{b x_{F}}^{\star} \mathbf{R} \pi_{2 \star}\left(\pi_{1}^{\star}(H) \otimes \mathcal{U}\right) \otimes x_{F}^{-d} .
\end{aligned}
$$

Proposition 1A. For a generic sheaf $V$ of fixed determinant and determinant of FourierMukai

$$
\operatorname{det} V=\mathcal{O}(d \sigma+m f), \operatorname{det} \widehat{V}=\mathcal{O}(-m \sigma-d f)
$$

we have

$$
\mathbf{R} \mathcal{S}^{\dagger}(V)=I_{Z}^{\vee} \otimes \mathcal{O}_{X}((a \chi+b m) f)
$$

for a subscheme $Z$ of length $d_{v}$ with $a(Z)=0$.

Proof. In the proof of this Proposition, it will be important to distinguish between the two copies of $X$ which are the source and the target of the Fourier-Mukai transform, because of the asymmetry present in the bundle $\mathcal{U}$. We will write $X_{1}$ and $X_{2}$ for two copies of $X$, respectively.

By Grothendieck duality, to prove that

$$
\mathbf{R} \mathcal{S}^{\dagger}(V)=I_{Z}^{\vee} \otimes \mathcal{O}_{X}((a \chi+b m) f)
$$

it suffices to show that

$$
\Psi_{X_{1} \rightarrow X_{2}}^{\mathcal{U}^{\vee}}\left(V^{\vee}\right)=I_{Z} \otimes \mathcal{O}(-(a \chi+b m) f)[-1],
$$

where $\Psi$ is the Fourier-Mukai transform with kernel $\mathcal{U}^{\vee}$. Using $[\mathrm{B}$, Lemma 6.4, we know that $V^{\vee}$ is $W I T_{1}$ with respect to $\Psi$, since the restriction to the general fiber is stable, of slope $-d / r<b / a$. Note that $\Psi\left(V^{\vee}\right)[1]$ has rank

$$
-\chi\left(\left.\left.V^{\vee}\right|_{F \times y} \otimes \mathcal{U}^{\vee}\right|_{F \times y}\right)=a d+b r=1 .
$$

Section 7 of [B], or Sections 3 and 5 in $[\mathrm{BH}]$, show that for generic $V$, the $\Psi$-transform is torsion-free, hence it must be of the form $I_{Z} \otimes L[-1]$ for some line bundle $L$. Bridgeland's argument moreover shows that the subscheme $Z$ has length $d_{v}$. 
The fiber degree of $\Psi\left(V^{\vee}\right)$ equals

$$
c_{1}\left(\mathbf{R} \pi_{2 \star}\left(\left.\pi_{1}^{\star} V\right|_{\mathrm{f}} ^{\vee} \otimes \mathcal{U}^{\vee}\right)\right)=\pi_{2 \star}\left(\pi_{1}^{\star}(r-d \omega)\left(a-c_{1}(\mathcal{U})+\operatorname{ch}_{2}(\mathcal{U})\right)\right)_{(2)}=0,
$$

where Lemmas 2 and 3 are used to express the numerical invariants of $\mathcal{U}$. In fact more is true. Since the restriction of $V$ to a generic fiber is stable, it must equal the Atiyah bundle $\mathrm{E}_{r, d}$. This implies that the restriction of $L$ to a generic fiber must coincide with $\Psi\left(\mathrm{E}_{r, d}\right)[1]$ which is trivial. Therefore, $L$ must be a sheaf of the form $\pi_{B}^{\star} M^{\vee}$, where $M$ is a degree $-\beta$ line bundle over $B$. We prove

$$
\beta=-a \chi-b m
$$

To this end, we calculate the Euler characteristic of $\Psi\left(V^{\vee}(\sigma)\right)$ as

$$
\begin{aligned}
\chi\left(\Psi\left(V^{\vee}\right)(\sigma)\right) & =\chi\left(F \times F \times B, \pi_{13}^{\star} V^{\vee} \otimes \pi_{12}^{\star} \mathcal{U}^{\vee} \otimes \pi_{23}^{\star} \mathcal{O}(\sigma)\right) \\
& =\int_{F \times F \times B} \pi_{13}^{\star}(r-(d \sigma+m f)+\chi \omega) \cdot \pi_{12}^{\star}\left(a-c_{1}(\mathcal{U})+\operatorname{ch}_{2}(\mathcal{U})\right) \cdot \pi_{23}^{\star}(1+\sigma) \\
& =b m+a \chi-\chi r+m d .
\end{aligned}
$$

On the other hand,

$$
\chi\left(I_{Z} \otimes L(\sigma)\right)=\beta-d_{v}=\beta-(d m-r \chi)
$$

hence $\beta=-a \chi-b m$.

When the determinant and determinant of Fourier-Mukai of $V$ are fixed, we show that $a(Z)=0$. First, we analyze the requirement the determinant be fixed. The inverse of $\mathbf{R} \mathcal{S}^{\dagger}$ is given by $\Phi_{X_{2} \rightarrow X_{1}}^{\mathcal{U}}$, the Fourier-Mukai whose kernel is $\mathcal{U}^{\vee}[1]$, considered as a transform from $X_{2} \rightarrow X_{1}$. Hence,

$$
V=\Phi_{X_{2} \rightarrow X_{1}}^{\mathcal{U}}\left(L^{\vee} \otimes I_{Z}^{\vee}\right), \quad L=\pi_{B}^{\star} M^{\vee}
$$

has fixed determinant $\mathcal{O}(d \sigma+m f)$. In order to make the computations more explicit, we write

$$
M=\mathcal{O}_{B}\left(-(\beta+1)\left[o_{B}\right]+[\mu]\right),
$$

for some $\mu \in B$. We have

$$
\begin{aligned}
\operatorname{det} V^{\vee} & =\operatorname{det} \Phi^{\mathcal{U} \vee}\left(L^{\vee}\right) \bigotimes_{z \in Z} \operatorname{det} \mathbf{R} \pi_{13 !}\left(\pi_{12}^{\star} \mathcal{U}^{\vee} \otimes \pi_{23}^{\star} \mathcal{O}_{z}^{\vee}\right)^{\vee} \\
& =\operatorname{det} \Phi^{\mathcal{U}^{\vee}}\left(L^{\vee}\right) \otimes \bigotimes \operatorname{det}\left(\left.\mathcal{U}^{\vee}\right|_{F \times z_{F}} \otimes \mathcal{O}_{z_{B}}[2]\right)^{\vee} \\
& =\operatorname{det} \Phi^{\mathcal{U}^{\vee}}\left(L^{\vee}\right) \otimes_{z \in Z}\left(\mathcal{O}_{F} \otimes \mathcal{O}_{B}\left(-a\left[z_{B}\right]\right)\right) \\
& =\operatorname{det} \Phi^{\mathcal{U}^{\vee}}\left(L^{\vee}\right) \otimes\left(\mathcal{O}_{F} \otimes \mathcal{O}_{B}\left(-\left(a d_{v}-1\right)\left[o_{B}\right]-\left[a \cdot a_{B}(Z)\right]\right)\right) \\
& =\mathcal{O}(-d \sigma-m f) \otimes \pi^{\star} \mathcal{O}_{B}\left(-\left[a \cdot a_{B}(Z)+r \mu\right]+\left[o_{B}\right]\right) .
\end{aligned}
$$


This gives

$$
a \cdot a_{B}(Z)+r \mu=o_{B}
$$

In equation (11), we used the calculation

$$
\begin{gathered}
\operatorname{det} \Phi^{\mathcal{U}^{\vee}}\left(L^{\vee}\right)=\operatorname{det} \mathbf{R} p_{13 !}\left(p_{12}^{\star} \mathcal{U}^{\vee} \otimes p_{3}^{\star} M\right)=\operatorname{det}\left(\mathbf{R} p_{1 !} \mathcal{U}^{\vee} \otimes M\right)=\operatorname{det} \mathbf{R} p_{1 !}\left(\mathcal{U}^{\vee}\right) \otimes M^{-r} \\
=\mathcal{O}_{F}\left(-d o_{F}\right) \otimes \mathcal{O}_{B}\left((r \beta+1)\left[o_{B}\right]-[r \mu]\right),
\end{gathered}
$$

where by Lemma 3 , the pushforward of $\mathcal{U}^{\vee}$ has rank $-r$ and degree $-d$. Equation (11) also makes use of the identity

$$
r \beta-a d_{v}=-m .
$$

We analyze the requirement that the determinant of the Fourier-Mukai be fixed. We know that

$$
\mathbf{R} \mathcal{S}(V)=\mathbf{R} \mathcal{S} \circ \Phi_{X_{2} \rightarrow X_{1}}^{\mathcal{U} \vee[1]}\left(L^{\vee} \otimes I_{Z}^{\vee}\right)
$$

This composition can be re-expressed as a Fourier-Mukai whose kernel equals the convolution of the following two kernels: $\widetilde{\mathcal{U}}^{\vee}[1]$ for $\Phi$, and

$$
\mathcal{P}=\mathcal{P}_{F} \times \mathcal{P}_{B}
$$

for $\mathbf{R} \mathcal{S}$. The tilde sign indicates that the kernel $\mathcal{U}^{\vee}[1]$ is considered in the opposite direction for $\Phi$ than it is for $\Psi$. This is the same as applying to $\mathcal{U} \rightarrow F \times F$ the involution that exchanges the factors. The new kernel can be expressed as

$$
\mathbf{R} p_{13 \star}\left(p_{12}^{\star} \widetilde{\mathcal{U}}^{\vee}[1] \otimes p_{23}^{\star} \mathcal{P}\right)=\mathbf{R} \pi_{13 \star}\left(\pi_{12}^{\star} \widetilde{\mathcal{U}}^{\vee} \otimes \pi_{23}^{\star} \mathcal{P}_{F}\right)[1] \otimes \mathcal{P}_{B}=\mathcal{V} \otimes \mathcal{P}_{B}
$$

where

$$
\mathcal{V} \rightarrow F \times F, \mathcal{V}=\mathbf{R} \pi_{13 \star}\left(\pi_{12}^{\star} \widetilde{\mathcal{U}}^{\vee} \otimes \pi_{23}^{\star} \mathcal{P}_{F}\right)[1]
$$

is the fiberwise Fourier-Mukai image of $\widetilde{\mathcal{U}}^{\vee}$ up to a shift. The complex $\mathcal{V}$ has rank $b$, and a Riemann-Roch calculation shows that

$$
c_{1}(\mathcal{V})=d\left[o_{F} \times F\right]+a\left[F \times o_{F}\right]+c_{1}\left(\mathcal{P}_{F}\right) .
$$

The pushforward $\mathbf{R}\left(p_{2}^{F}\right)_{\star}(\mathcal{V})$ has rank $d$ and determinant $-r\left[o_{F}\right]$. This will be used below. Fiberwise, note that

$$
\left.\operatorname{det} \mathcal{V}^{\vee}\right|_{z_{F} \times F}=\operatorname{det} \mathbf{R} p_{2 !}\left(\left.p_{1}^{\star} \widetilde{\mathcal{U}}\right|_{z_{F} \times F} ^{\vee} \otimes \mathcal{P}_{F}\right)=-\left[z_{F}\right]-(a-1)\left[o_{F}\right] .
$$

Now, we calculate

$$
\begin{aligned}
\operatorname{det} \mathbf{R} \mathcal{S}(V) & =\operatorname{det} \mathbf{R} p_{2 \star}\left(\mathcal{V} \otimes \mathcal{P}_{B} \otimes p_{1}^{\star}\left(L^{\vee} \otimes I_{Z}^{\vee}\right)\right) \\
& =\operatorname{det} \mathbf{R} p_{2 \star}\left(\mathcal{V} \otimes \mathcal{P}_{B} \otimes p_{1}^{\star} L^{\vee}\right) \otimes \operatorname{det} \mathbf{R} p_{2 \star}\left(\mathcal{V} \otimes \mathcal{P}_{B} \otimes p_{1}^{\star} \mathcal{O}_{Z}[2]\right)^{\vee}
\end{aligned}
$$

The first determinant is constant

$$
\operatorname{det} \mathbf{R} p_{2 \star}\left(\mathcal{V} \otimes \mathcal{P}_{B} \otimes p_{1}^{\star} L^{\vee}\right)=\operatorname{det}\left(\mathbf{R}\left(p_{2}^{F}\right)_{\star}(\mathcal{V}) \otimes \mathbf{R}\left(p_{2}^{B}\right)_{\star}\left(\mathcal{P}_{B} \otimes\left(p_{1}^{B}\right)^{\star} M\right)\right)
$$




$$
=\left(\operatorname{det} \mathbf{R}\left(p_{2}^{F}\right)_{\star}(\mathcal{V})\right)^{-\beta} \otimes \mathcal{O}_{B}(-[-\mu])^{d}=\mathcal{O}_{F}\left(r \beta\left[o_{F}\right]\right) \otimes \mathcal{O}_{B}(-d[-\mu]) .
$$

The second determinant needs to be fixed, and it equals

$$
\begin{gathered}
\left.\bigotimes_{z \in Z} \operatorname{det} \mathcal{V}^{\vee}\right|_{z_{F} \times F} \otimes \mathcal{O}_{B}\left(-\left[z_{B}\right]+\left[o_{B}\right]\right)^{b}=\bigotimes_{z \in Z} \mathcal{O}_{F}\left(-\left[z_{F}\right]-(a-1)\left[o_{F}\right]\right) \otimes \mathcal{O}_{B}\left(-\left[z_{B}\right]+\left[o_{B}\right]\right)^{b} \\
=\mathcal{O}_{F}\left(-\left[a_{F}(Z)\right]-\left(a d_{v}-1\right)\left[o_{F}\right]\right) \otimes \mathcal{O}_{B}\left(-\left[b \cdot a_{B}(Z)\right]+\left[o_{B}\right]\right) .
\end{gathered}
$$

Therefore

$$
\operatorname{det} \mathbf{R} \mathcal{S}(V)=\mathcal{O}(-m f-d \sigma) \otimes \mathcal{O}_{F}\left(-\left[a_{F}(Z)\right]+\left[o_{F}\right]\right) \otimes \mathcal{O}_{B}\left(-\left[b \cdot a_{B}(Z)-d \mu\right]+\left[o_{B}\right]\right) .
$$

Since

$$
\operatorname{det} \mathbf{R} \mathcal{S}(V)=\mathcal{O}(-m f-d \sigma)
$$

this immediately yields

$$
a_{F}(Z)=o_{F} \text { and } b \cdot a_{B}(Z)-d \mu=o_{B} .
$$

Combining these two equations with

$$
a \cdot a_{B}(Z)+r \mu=o_{B}
$$

shown aprove, and the fact that $a d+b r=1$, we obtain $a_{B}(Z)=0$ and $\mu=0$. Thus

$$
a(Z)=0, L=\mathcal{O}((a \chi+b m) f) .
$$

This completes the proof.

Proof of Theorem 1. In the course of the proof, we showed that for a generic sheaf $V$ of rank $r$ and determinant

$$
\operatorname{det} V=\mathcal{O}(d \sigma+m f),
$$

the Fourier-Mukai transform takes the form

$$
\mathbf{R} \mathcal{S}^{\dagger}(V)=I_{Z}^{\vee}((a \chi+b m) f) \otimes \pi_{B}^{\star} \mu,
$$

for some $\mu \in B \cong \widehat{B}$ such that

$$
a \cdot a_{B}(Z)+r \mu=o_{B} .
$$

The assignment

$$
V \rightarrow\left(Z, b a_{B}(Z)-d \mu\right)
$$

gives the birational isomorphism

$$
\mathfrak{M}_{v}^{+} \rightarrow \mathfrak{X}_{v}^{+}
$$

claimed by Theorem 1 . 
Remark 2. In a similar fashion we could find the birational type of the moduli space $\mathfrak{M}_{v}^{-}$. However, it is not necessary to repeat the argument above to deal with this new case. We could instead make use of the map

$$
\Phi_{v}^{-}: K_{v} \times X \rightarrow \mathfrak{M}_{v}^{-},(E, y) \mapsto t_{\left(d y_{B}, m y_{F}\right)}^{\star} E \otimes y^{\chi}
$$

and use Lemma $1 \mathrm{~A}$ to calculate

$$
\begin{aligned}
\mathbf{R S}^{\dagger}\left(\Phi_{v}^{-}(E, y)\right) & =\mathbf{R} \mathcal{S}^{\dagger}\left(t_{\left(d y_{B}, m y_{F}\right)}^{\star} E \otimes y^{\chi}\right)=t_{a \chi y_{F}}^{\star} \mathbf{R} \mathcal{S}^{\dagger}\left(t_{\left(d y_{B}, m y_{F}\right)}^{\star} E\right) \otimes\left(y_{B}^{\chi} \otimes y_{F}^{r \chi}\right) \\
& =t_{a \chi y_{F}}^{\star}\left(t_{d y_{B}+b m y_{F}}^{\star} \mathbf{R} \mathcal{S}^{\dagger}(E) \otimes y_{F}^{-d m}\right) \otimes\left(y_{B}^{\chi} \otimes y_{F}^{r \chi}\right) \\
& =t_{d y_{B}+(a \chi+b m) y_{F}}^{\star}\left(I_{Z}^{\vee}((a \chi+b m) f)\right) \otimes\left(y_{B}^{\chi} \otimes y_{F}^{-d_{v}}\right) \\
& =I_{Z^{-}}^{\vee}((a \chi+b m) f) \otimes\left(y_{B}^{\chi-(a \chi+b m) d} \otimes y_{F}^{-d_{v}}\right) \\
& =I_{Z^{-}}^{\vee}((a \chi+b m) f) \otimes\left(y_{B}^{-b d_{v}} \otimes y_{F}^{-d_{v}}\right)
\end{aligned}
$$

where $Z^{-}=t_{d y_{B}+(a \chi+b m) y_{F}}^{\star} Z$. Thus, the assignment

$$
V \mapsto\left(Z^{-},-d_{v} y\right)
$$

gives a birational isomorphism

$$
\mathfrak{M}_{v}^{-}-\rightarrow \mathfrak{X}_{v}^{-}=\left\{\left(Z^{-}, z\right): a\left(Z^{-}\right)=f(z)\right\} \hookrightarrow X^{\left[d_{v}\right]} \times X
$$

where the isogeny $f: X \rightarrow X$ is given by

$$
f(z)=\left(d z_{B},(a \chi+b m) z_{F}\right) .
$$

In the case of fiber degree 1 , this specializes to the subvariety

$$
\mathfrak{X}_{v}^{-}=\left\{\left(Z^{-}, z_{F}\right): a_{F}\left(Z^{-}\right)=\chi z_{F}\right\} \hookrightarrow X^{\left[d_{v}\right]} \times F
$$

of Section 2 ,

3.2. Higher genus. Assume now $(C, o)$ is a pointed smooth curve of genus $g \geq 1$, and $F$ is still an elliptic curve. We set $\bar{g}=g-1$. Consider the product surface

$$
X=C \times F \rightarrow C .
$$

Let $\mathfrak{M}_{v}^{+}$be the moduli space of sheaves over $X$ of rank $r$ and determinant $\mathcal{O}(\sigma+m f)$, where $\sigma$ is the zero section and $f$ denotes the fiber over $o$. We describe the birational type of $\mathfrak{M}_{v}^{+}$, in codimension 1 for $r \neq 2$, using the Fourier-Mukai transform with kernel the Poincaré bundle

$$
\pi_{F \times F}^{\star} \mathcal{P}_{F} \rightarrow F \times F \times C .
$$

The proof is entirely similar to that of Proposition 1A, so we content here to only record the result. 
Let

$$
a_{C}: X^{\left[d_{v}\right]} \rightarrow C^{\left[d_{v}\right]}
$$

be the map induced by the projection $X \rightarrow C$. Thus, each scheme $Z$ of length $d_{v}$ in $X$ yields a divisor $a_{C}(Z)$ of degree $d_{v}$ over the curve $C$. The line bundle

$$
\mathcal{M}_{Z}=\mathcal{O}_{C}\left(a_{C}(Z)-d_{v} \cdot o\right)
$$

has degree 0 , and therefore admits roots of order $r$. We define

$$
\mathfrak{X}_{v}^{+}=\left\{(Z, c): c^{r}=\mathcal{M}_{Z}\right\} \hookrightarrow X^{\left[d_{v}\right]} \times \operatorname{Pic}^{0}(C) .
$$

Then, for $V \in \mathfrak{M}_{v}^{+}$, we have

$$
\mathbf{R} \mathcal{S}^{\dagger}(V)=I_{Z}^{\vee}(-r \sigma+(\chi+\bar{g}) f) \otimes c^{-1},
$$

establishing the birational isomorphism

$$
\mathfrak{M}_{v}^{+} \rightarrow \mathfrak{X}_{v}^{+}
$$

The same statement holds in any fiber degree coprime to the rank, but we will not detail this fact.

\section{The STRAnge DUALity ISOMORPhism}

We now proceed to prove Theorems 2 , 3 and 2A stated in the introduction. Throughout this section, we place ourselves in the context when the fiber degree is 1 .

4.1. Reformulation. Let $X=B \times F$ be a product abelian surface. As a consequence of Section 2, under the birational map

$$
\mathfrak{M}_{v}^{+} \times \mathfrak{M}_{w}^{+} \rightarrow \mathfrak{X}_{v}^{+} \times \mathfrak{X}_{w}^{+}
$$

induced by the relative Fourier-Mukai transform, the standard theta divisor

$$
\Theta_{v w}^{+}=\left\{(V, W) \text { with } h^{1}(V \otimes W) \neq 0\right\} \subset \mathfrak{M}_{v}^{+} \times \mathfrak{M}_{w}^{+}
$$

is identified with a divisor

$$
\Theta^{+} \subset \mathfrak{X}_{v}^{+} \times \mathfrak{X}_{w}^{+} .
$$

Note that for sheaves $(V, W) \in \mathfrak{M}_{v}^{+} \times \mathfrak{M}_{w}^{+}$corresponding to pairs

$$
\left(Z^{+}, z_{B}\right) \in \mathfrak{X}_{v}^{+} \text {and }\left(T^{+}, t_{B}\right) \in \mathfrak{X}_{w}^{+},
$$


we have

$$
\begin{aligned}
H^{1}(V \otimes W) & =\operatorname{Ext}^{1}\left(W^{\vee}, V\right)=\operatorname{Ext}^{1}\left(\mathbf{R S}^{\dagger}\left(W^{\vee}\right), \mathbf{R} \mathcal{S}^{\dagger}(V)\right) \\
& =\operatorname{Ext}^{1}\left(I_{\widetilde{T^{+}}}\left(s \sigma-\chi^{\prime} f\right)[-1] \otimes t_{B}, I_{Z^{+}}^{\vee} \otimes \mathcal{O}(-r \sigma+\chi f) \otimes z_{B}^{-1}\right) \\
& =\operatorname{Ext}^{1}\left(I_{Z^{+}}^{\vee} \otimes \mathcal{O}(-r \sigma+\chi f) \otimes z_{B}^{-1}, I_{\widetilde{T^{+}}}\left(s \sigma-\chi^{\prime} f\right)[-1] \otimes t_{B}\right)^{\vee} \\
& =H^{0}\left(I_{Z^{+}} \otimes I_{\widetilde{T^{+}}} \otimes z_{B} \otimes t_{B} \otimes \mathcal{O}\left((r+s) \sigma-\left(\chi+\chi^{\prime}\right) f\right)\right)^{\vee} .
\end{aligned}
$$

(The notation above has the obvious meaning: $r, s$ are the ranks of $v$ and $w$, while $\chi, \chi^{\prime}$ are their Euler characteristics.) Thus, the theta divisor $\Theta_{v w}^{+}$in the product $\mathfrak{M}_{v}^{+} \times \mathfrak{M}_{w}^{+}$ corresponds to the divisor

$$
\Theta^{+}=\left\{\left(Z^{+}, z_{B}, T^{+}, t_{B}\right): h^{0}\left(I_{Z^{+}} \otimes I_{\widetilde{T^{+}}} \otimes z_{B} \otimes t_{B} \otimes L\right) \neq 0\right\}
$$

in the product $\mathfrak{X}_{v}^{+} \times \mathfrak{X}_{w}^{+}$. Here, we set

$$
L=\mathcal{O}\left((r+s) \sigma-\left(\chi+\chi^{\prime}\right) f\right) \text { on } X .
$$

In consequence, strange duality is demonstrated if we show that the divisor $\Theta^{+}$induces an isomorphism

$$
\mathrm{D}^{+}: H^{0}\left(\mathfrak{X}_{v}^{+}, \Theta_{w}\right)^{\vee} \longrightarrow H^{0}\left(\mathfrak{X}_{w}^{+}, \Theta_{v}\right)
$$

4.2. Theta bundles. Since for $r \neq 2$ the birational isomorphism $\mathfrak{M}_{v}^{+--\rightarrow} \mathfrak{X}_{v}^{+}$is defined away from codimension 2, we are interested in an explicit description of the determinant line bundle

$$
\Theta_{w} \rightarrow \mathfrak{X}_{v}^{+}
$$

For a line bundle $L$ on $X$, we standardly let

$$
L^{\left[d_{v}\right]}=\operatorname{det} R p_{\star}\left(\mathcal{O}_{\mathcal{Z}} \otimes q^{\star} L\right) \text { on } X^{\left[d_{v}\right]} .
$$

We also note the natural projections

$$
c_{v}: \mathfrak{X}_{v}^{+} \rightarrow X^{\left[d_{v}\right]},\left(Z^{+}, z_{B}\right) \mapsto Z^{+}
$$

and

$$
\pi_{2}: \mathfrak{X}_{v}^{+} \rightarrow B,\left(Z^{+}, z_{B}\right) \rightarrow z_{B} .
$$

The theta bundle is calculated by the following result:

Proposition 2. We have

$$
\Theta_{w}=c_{v}^{\star} L^{\left[d_{v}\right]} \otimes \pi_{2}^{\star} \mathcal{O}_{B}\left((s-r) o_{B}\right),
$$

with $L$ given by (12). 
Proof. We give one proof of the proposition here; another one is essentially contained in Section 4.4. We begin by noting the degree $d_{v}^{4}$ étale morphism

$$
q_{v}: K^{\left[d_{v}\right]} \times X=K^{\left[d_{v}\right]} \times B \times F \rightarrow \mathfrak{X}_{v}^{+}, \quad(Z, x) \mapsto\left(t_{r x_{B}+x_{F}}^{\star} Z,-d_{v} x_{B}\right)
$$

It is related to the standard less twisted map

$$
\mu_{v}: K^{\left[d_{v}\right]} \times X \rightarrow X^{\left[d_{v}\right]}, \quad \mu_{v}(Z, x)=t_{x}^{\star} Z
$$

via the commutative diagram

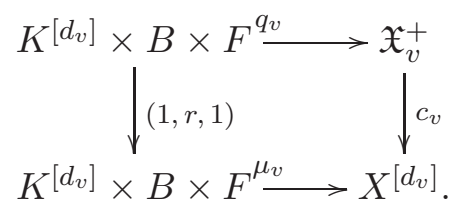

Using the diagram, we calculate

$$
q_{v}^{\star} c_{v}^{\star} L^{\left[d_{v}\right]}=(1, r, 1)^{\star} \mu_{v}^{\star} L^{\left[d_{v}\right]}=(1, r, 1)^{\star}\left(L^{\left[d_{v}\right]} \otimes L^{d_{v}}\right)=L^{\left[d_{v}\right]} \otimes\left((r, 1)^{\star} L\right)^{d_{v}},
$$

and further,

$$
(r, 1)^{\star} L=(r, 1)^{\star} \mathcal{O}\left((r+s) \sigma-\left(\chi+\chi^{\prime}\right) f\right)=\mathcal{O}\left((r+s) \sigma-r^{2}\left(\chi+\chi^{\prime}\right) f\right) .
$$

If

$$
\pi_{B}: K^{\left[d_{v}\right]} \times B \times F \rightarrow B
$$

is the projection to $B$, from the definitions we also have $\pi_{2} \circ q_{v}=-d_{v}$ hence

$$
q_{v}^{\star} \pi_{2}^{\star} \mathcal{O}_{B}\left((s-r) o_{B}\right)=\pi_{B}^{\star} \mathcal{O}_{B}\left((s-r) d_{v}^{2} o_{B}\right) .
$$

Putting the previous three equations together we find

$$
q_{v}^{\star}\left(c_{v}^{\star} L^{\left[d_{v}\right]} \otimes \pi_{2}^{\star} \mathcal{O}_{B}\left((s-r) o_{B}\right)\right)=L^{\left[d_{v}\right]} \otimes \mathcal{O}\left((r+s) \sigma+\left((s-r) d_{v}-r^{2}\left(\chi+\chi^{\prime}\right)\right) f\right)^{\otimes d_{v}} .
$$

On the other hand, Proposition 2 of [MO2] (written in holomorphic $K$-theory, as in [0]) gives

$$
q_{v}^{\star} \Theta_{w}=L^{\left[d_{v}\right]} \otimes \mathcal{O}((r+s) \sigma+(r n+s m) f)^{\otimes d_{v}} .
$$

The two pullbacks (13) and (14) are seen equal on $K^{\left[d_{v}\right]} \times X$, as one shows that

$$
r n+s m=(s-r) d_{v}-r^{2}\left(\chi+\chi^{\prime}\right) .
$$

This uses the numerical identity

$$
m+n=-r \chi^{\prime}-s \chi
$$

which expresses strange duality orthogonality $\chi(v \cdot w)=0$, also remembering that $d_{v}=$ $m-r \chi$. 
Now, consider

$$
Q=\Theta_{w} \otimes\left(c_{v}^{\star} L^{\left[d_{v}\right]}\right)^{\vee} \otimes \pi_{B}^{\star} \mathcal{O}_{B}\left((r-s) o_{B}\right) .
$$

We showed that $q_{v}^{\star} Q$ is trivial. Note the morphism

$$
p_{v}: \mathfrak{X}_{v}^{+} \rightarrow X,\left(Z, x_{B}\right) \rightarrow\left(a_{F}(Z), x_{B}\right)
$$

with fibers isomorphic to $K^{\left[d_{v}\right]}$. In fact, each fiber

$$
\iota: p_{v}^{-1}(x) \hookrightarrow \mathfrak{X}_{v}^{+}
$$

factors through the morphism

$$
q_{v}: K^{\left[d_{v}\right]} \times X \rightarrow \mathfrak{X}_{v}^{+} .
$$

Indeed, $\iota=q_{v} \circ j$, where $j: p_{v}^{-1}(x) \rightarrow K^{\left[d_{v}\right]} \times X$ is the map

$$
j(Z)=\left(t_{-r y_{B}-y_{F}}^{\star} Z, y\right),
$$

for any choice of $y \in X$ such that $d_{v} y=-x$. Therefore, the above argument implies that the restriction of $Q$ to each fiber $p_{v}^{-1}(x)$ is trivial. Hence,

$$
Q=p_{v}^{\star} N
$$

for some line bundle $N$ over $X$.

We now argue that $N$ is trivial, by constructing a suitable test family. Consider $Z_{0}$ a subscheme of length $d_{v}-1$ supported at 0 , and define

$$
\alpha: X \rightarrow \mathfrak{X}_{v}^{+}, x \mapsto\left(Z_{0}+\left(r x_{B}, x_{F}\right), x_{B}\right) .
$$

The map $\alpha$ is defined away from the $r^{2}$ points in $B[r] \times o_{F}$. It suffices to show $N$ is trivial along this open set. Pulling back the equality

$$
p_{v}^{\star} N=\Theta_{w} \otimes\left(c_{v}^{\star} L^{\left[d_{v}\right]}\right)^{\vee} \otimes \pi_{B}^{\star} \mathcal{O}_{B}\left((r-s) o_{B}\right)
$$

under $\alpha$, and noting $\alpha \circ p_{v}=1$, it suffices to prove that

$$
\alpha^{\star} \Theta_{w}=\bar{c}_{v}^{\star} L^{\left[d_{v}\right]} \otimes \pi_{B}^{\star} \mathcal{O}_{B}\left((s-r) o_{B}\right)
$$

where

$$
\bar{c}_{v}=c_{v} \circ \alpha: X \rightarrow X^{\left[d_{v}\right]} \text { is given by } \bar{c}_{v}(x)=Z_{0}+\left(r x_{B}, x_{F}\right) .
$$

We calculate the right hand side. The universal family

$$
\mathcal{Z} \subset X^{\left[d_{v}\right]} \times X
$$

becomes under pullback by $\bar{c}_{v}$ the family $Z_{0}+\Delta_{r} \cong \Delta_{r}$, where $\Delta_{r} \subset X \times X$ is the $r$-fold diagonal

$$
\Delta_{r}=\left(r x_{B}, x_{F}, x_{B}, x_{F}\right) .
$$


Then,

$$
\bar{c}_{v}^{\star} L^{\left[d_{v}\right]}=\bar{c}_{v}^{\star} \operatorname{det} \mathbf{R} p_{!}\left(\mathcal{O}_{\mathcal{Z}} \otimes q^{\star} L\right)=\operatorname{det} \mathbf{R} p_{!}\left(\mathcal{O}_{\Delta_{r}} \otimes q^{\star} L\right)=\left(r_{B}, 1_{F}\right)^{\star} L
$$

hence

$$
\bar{c}_{v}^{\star} L^{\left[d_{v}\right]}=\mathcal{O}\left((r+s) \sigma-r^{2}\left(\chi+\chi^{\prime}\right) f\right) .
$$

For the left hand side, fix $(T, c)$ in $\mathfrak{X}_{w}^{+}$, where $c \in B \cong \widehat{B}$, so that

$$
a_{B}(T)=s c
$$

We have

$$
\Theta_{w}=\operatorname{det} \mathbf{R} p_{!}\left(I_{\mathcal{Z}} \otimes \mathcal{P}_{B} \otimes q^{\star}\left(L \otimes c \otimes I_{\widetilde{T}}\right)\right)^{\vee},
$$

where $\mathcal{P}_{B}$ is the Poincaré bundle over $B \times B$. Write

$$
M=L \otimes c \otimes I_{\widetilde{T}}
$$

so that

$$
M=M_{B} \otimes M_{F}-\sum_{t \in T} \mathcal{O}_{t_{B} \times-t_{F}}
$$

for

$$
M_{B}=c \otimes \mathcal{O}_{B}\left(-\left(\chi+\chi^{\prime}\right) o_{B}\right), M_{F}=\mathcal{O}\left((r+s) o_{F}\right) .
$$

Since the universal family pulls back to $\alpha^{\star} \mathcal{Z} \cong \Delta_{r}$, we have that

$$
\alpha^{\star} I_{\mathcal{Z}}=\mathcal{O}-\mathcal{O}_{\Delta_{r}}
$$

Therefore,

$$
\begin{gathered}
\alpha^{\star} \Theta_{w}=\operatorname{det} \mathbf{R} p_{!}\left(\mathcal{P}_{B} \otimes q^{\star}\left(M_{B} \otimes M_{F}\right)\right)^{\vee} \otimes \operatorname{det} \mathbf{R} p_{!}\left(\mathcal{O}_{\Delta_{r}} \otimes \mathcal{P}_{B} \otimes q^{\star}\left(M_{B} \otimes M_{F}\right)\right) \\
\otimes_{t \in T}\left(\operatorname{det} \mathbf{R} p_{!}\left(\mathcal{P}_{B} \otimes q^{\star} \mathcal{O}_{t_{B} \times-t_{F}}\right) \otimes \operatorname{det} \mathbf{R} p_{!}\left(\mathcal{O}_{\Delta_{r}} \otimes \mathcal{P}_{B} \otimes q^{\star} \mathcal{O}_{t_{B} \times-t_{F}}\right)^{\vee}\right)
\end{gathered}
$$

We calculate the first term

$$
\begin{gathered}
\operatorname{det} \mathbf{R} p_{!}\left(\mathcal{P}_{B} \otimes q^{\star}\left(M_{B} \otimes M_{F}\right)\right)^{\vee}=\operatorname{det}\left(\mathbf{R} p_{B !}\left(\mathcal{P}_{B} \otimes q_{B}^{\star} M_{B}\right) \otimes H^{\bullet}\left(M_{F}\right) \otimes \mathcal{O}_{F}\right)^{\vee} \\
=\operatorname{det}\left(\mathbf{R} p_{B !}\left(\mathcal{P}_{B} \otimes q_{B}^{\star} M_{B}\right)\right)^{-(r+s)} \otimes \mathcal{O}_{F}=\left(c \otimes \mathcal{O}_{B}\left(-o_{B}\right)\right)^{-(r+s)} \otimes \mathcal{O}_{F} .
\end{gathered}
$$

The second term becomes

$$
\operatorname{det} \mathbf{R} p_{!}\left(\mathcal{O}_{\Delta_{r}} \otimes \mathcal{P}_{B} \otimes q^{\star}\left(M_{B} \otimes M_{F}\right)\right)=\operatorname{det}\left(\mathbf{R} p_{B !}\left(\mathcal{O}_{\Delta_{r}^{B}} \otimes \mathcal{P}_{B} \otimes q_{B}^{\star} M_{B}\right) \otimes M_{F}\right)
$$

where $\Delta_{r}^{B}$ is the image of

$$
j: B \rightarrow \Delta_{r}^{B}, x_{B} \rightarrow\left(r x_{B}, x_{B}\right)
$$

We calculate

$$
\mathbf{R} p_{B !}\left(\mathcal{O}_{\Delta_{r}^{B}} \otimes\left(\mathcal{P}_{B} \otimes q_{B}^{\star} M_{B}\right)\right)=j^{\star} \mathcal{P}_{B} \otimes r^{\star} M_{B}=\mathcal{O}_{B}\left(-2 r o_{B}\right) \otimes r^{\star} M_{B}
$$


Therefore, the second term equals

$$
c^{r} \otimes \mathcal{O}_{B}\left(-2 r o_{B}-r^{2}\left(\chi+\chi^{\prime}\right) o_{B}\right) \otimes \mathcal{O}_{F}\left((r+s) o_{F}\right) .
$$

The third term now equals

$$
\operatorname{det} \mathbf{R} p_{!}\left(\mathcal{P}_{B} \otimes q^{\star} \mathcal{O}_{t_{B} \times-t_{F}}\right)=\mathcal{P}_{t_{B}} \otimes \mathcal{O}_{F},
$$

hence the tensor product over all $t \in T$ yields

$$
\mathcal{P}_{a_{B}(W)} \otimes \mathcal{O}_{F}=c^{s} \otimes \mathcal{O}_{F}
$$

Finally, the fourth term is easily seen to be trivial

$$
\operatorname{det} \mathbf{R} p_{!}\left(\mathcal{O}_{\Delta_{r}} \otimes \mathcal{P}_{B} \otimes q^{\star} \mathcal{O}_{t_{B} \times-t_{F}}\right)=\mathcal{O}_{X} .
$$

Equation (15) follows putting all the terms together. This concludes the proof of Proposition 2 ,

4.2.1. Fixed determinant of Fourier-Mukai. The discussion for the moduli space of sheaves with fixed determinant of their Fourier-Mukai is entirely parallel. We identify the theta divisor

$$
\Theta_{v w}^{-} \subset \mathfrak{M}_{v}^{-} \times \mathfrak{M}_{w}^{-}
$$

with the divisor

$$
\Theta^{-}=\left\{\left(Z^{-}, z_{F}, T^{-}, t_{F}\right): h^{0}\left(I_{Z^{-}} \otimes I_{\widetilde{T}^{-}} \otimes z_{F}^{-1} \otimes t_{F} \otimes L\right) \neq 0\right\} \hookrightarrow \mathfrak{X}_{v}^{-} \times \mathfrak{X}_{w}^{-},
$$

where as before

$$
L=\mathcal{O}\left(-\left(\chi+\chi^{\prime}\right) f+(r+s) \sigma\right)
$$

For $r, s \geq 3$, the birational isomorphisms are defined in codimension 1 , and we have

$$
\Theta_{w}=\left(c_{v}^{-}\right)^{\star} L^{\left[d_{v}\right]} \otimes \pi_{F}^{\star} \mathcal{O}_{F}\left(\left(\chi-\chi^{\prime}\right) o_{F}\right),
$$

where

$$
c_{v}^{-}: \mathfrak{X}_{v}^{-} \rightarrow X^{\left[d_{v}\right]}
$$

is the forgetful morphism.

4.3. Equal ranks and the proof of strange duality. We now consider the case $r=s \geq 3$, when we simply have

$$
\Theta_{w}=c_{v}^{\star} L^{\left[d_{v}\right]}, \Theta_{v}=c_{w}^{\star} L^{\left[d_{w}\right]} .
$$

Furthermore, tensor product gives a rational map defined away from codimension 2

$$
\tau^{+}: \mathfrak{X}_{v}^{+} \times \mathfrak{X}_{w}^{+} \rightarrow \mathfrak{X}^{+}\left(I_{Z}, z_{B}, I_{T}, t_{B}\right) \mapsto\left(I_{Z} \otimes I_{\widetilde{T}}, z_{B} \otimes t_{B}\right) .
$$


Here

$$
\mathfrak{X}^{+}=\left\{\left(U, u_{B}\right): a_{B}(U)=r u_{B}\right\} \subset X^{\left[d_{v}+d_{w}\right]} \times B .
$$

In other words, $\mathfrak{X}^{+}$is the fiber product

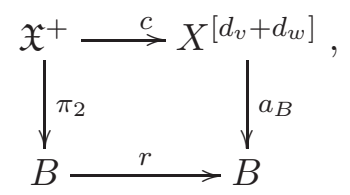

where $c$ and $\pi_{2}$ are the natural projection maps,

$$
c\left(U, u_{B}\right)=U, \pi_{2}\left(U, u_{B}\right)=u_{B} .
$$

The divisor

$$
\Theta^{+} \hookrightarrow \mathfrak{X}_{v}^{+} \times X_{w}^{+}
$$

is the pullback under $\tau^{+}$of the divisor

$$
\theta^{+}=\left\{\left(U, u_{B}\right): h^{0}\left(I_{U} \otimes u_{B} \otimes L\right) \neq 0\right\}
$$

with corresponding line bundle

$$
\mathcal{O}\left(\theta^{+}\right) \simeq c^{\star} L^{\left[d_{v}+d_{w}\right]} \text { on } \mathfrak{X}^{+} .
$$

To identify the space of sections $H^{0}\left(\mathfrak{X}^{+}, \mathcal{O}\left(\theta^{+}\right)\right)$, we let $B[r]$ denote the group of $r$-torsion points on $B$, and fix an isomorphism

$$
r_{\star} \mathcal{O} \simeq \oplus_{\tau \in B[r]} \tau .
$$

Then

$$
\begin{aligned}
& H^{0}\left(\mathfrak{X}^{+}, \mathcal{O}\left(\theta^{+}\right)\right)=H^{0}\left(\mathfrak{X}^{+}, c^{\star} L^{\left[d_{v}+d_{w}\right]}\right)=H^{0}\left(X^{\left[d_{v}+d_{w}\right]}, L^{\left[d_{v}+d_{w}\right]} \otimes(c)_{\star} \mathcal{O}\right)= \\
& =\bigoplus_{\tau \in B[r]} H^{0}\left(X^{\left[d_{v}+d_{w}\right]}, L^{\left[d_{v}+d_{w}\right]} \otimes a_{B}^{\star} \tau\right)=\bigoplus_{\tau \in B[r]} H^{0}\left(X^{\left[d_{v}+d_{w}\right]},(L \otimes \tau)^{\left[d_{v}+d_{w}\right]}\right) .
\end{aligned}
$$

Under the isomorphism above, the divisor $\theta^{+}$corresponds up to a $\mathbb{C}^{\star}$-scaling ambiguity to a tuple of sections,

$$
\theta^{+} \longleftrightarrow\left(s_{\tau}\right)_{\tau \in B[r]}, \quad s_{\tau} \in H^{0}\left(X^{\left[d_{v}+d_{w}\right]},(L \otimes \tau)^{\left[d_{v}+d_{w}\right]}\right) .
$$

The space of sections of $(L \otimes \tau)^{\left[d_{v}+d_{w}\right]} \rightarrow X^{\left[d_{v}+d_{w}\right]}$ can be identified with

$$
\Lambda^{d_{v}+d_{w}} H^{0}(L \otimes \tau)
$$

which is one dimensional since $h^{0}(L \otimes \tau)=\chi(L \otimes \tau)=d_{v}+d_{w}$. Furthermore, any non-zero section vanishes along the divisor

$$
\Theta_{L \otimes \tau}=\left\{I_{U}: \quad h^{0}\left(I_{U} \otimes L \otimes \tau\right) \neq 0\right\} .
$$

We have the important 
Proposition 3. For each $\tau, s_{\tau}$ is not the trivial section, hence it vanishes along $\Theta_{L \otimes \tau}$.

The proposition completes the proof of Theorem 2. Indeed, as explained in [MO1], each of the sections $s_{\tau}$ induces an isomorphism between spaces of sections

$$
\bigoplus_{\tau \in B[r]} s_{\tau}: \bigoplus_{\tau \in B[r]} H^{0}\left(X^{\left[d_{v}\right.},(L \otimes \tau)^{\left[d_{v}\right]}\right)^{\vee} \rightarrow \bigoplus_{\tau \in B[r]} H^{0}\left(X^{\left[d_{w}\right]},(L \otimes \tau)^{\left[d_{w}\right]}\right)
$$

Since we argued that the strange duality map coincides with $\bigoplus_{\tau \in B[r]} s_{\tau}$, Theorem 2 follows. 3

The proof of Theorem 3 is identical.

Proof of the Proposition. This is easily seen by restriction to $X_{F}^{\left[d_{v}+d_{w}\right]}$, the fiber over zero of the addition map

$$
a_{B}: X^{\left[d_{v}+d_{w}\right]} \rightarrow B
$$

Letting $\mathfrak{X}_{F}^{+}$be the fiber product

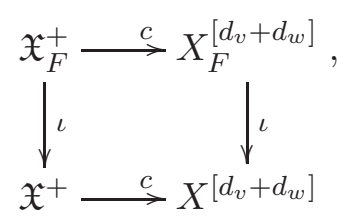

we have a commutative diagram

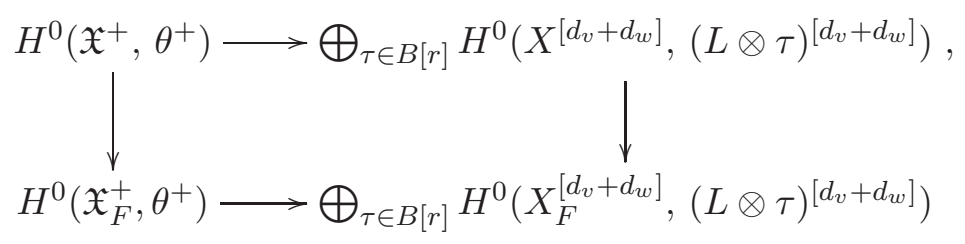

where the horizontal maps are isomorphisms and the vertical maps are restrictions of sections. The bottom isomorphism is particularly easy to understand since

$$
\mathfrak{X}_{F}^{+} \simeq X_{F}^{\left[d_{v}+d_{w}\right]} \times B[r] .
$$

Let $\theta_{F}^{+}$be the restriction of $\theta^{+}$to $\mathfrak{X}_{F}^{+}$. Further restricting $\theta_{F}^{+}$to $X_{F}^{\left[d_{v}+d_{w}\right]} \times\{\tau\}$ we obtain the divisor $\Theta_{L \otimes \tau}$. We claim that each of the divisors $\Theta_{L \otimes \tau}$ restricts nontrivially to $X_{F}^{\left[d_{v}+d_{w}\right]}$. This implies in turn that $s_{\tau}$ is not the trivial section for any $\tau$. The following lemma proves the claim above when $\tau=\mathcal{O}$; the arguments are identical for $\tau \neq \mathcal{O}$.

\footnotetext{
${ }^{3}$ Here, we assumed the determinant is $\mathcal{O}(\sigma+m f)$ where $f$ denotes the fiber over zero. If the determinant involves the fiber over a different point the same argument applies with the obvious changes in the definition of $L$.
} 
Lemma 4. Consider the line bundle $L_{k, n}=\mathcal{O}(k \sigma+n f)$ on $X=B \times F$, and assume that $k \geq 2, n \geq 1$. Then for a generic $I_{Z} \in X_{F}^{[k n]}$, we have

$$
H^{0}\left(I_{Z} \otimes L_{k, n}\right)=0 .
$$

Proof. We argue by induction on $n$. For $n=1$, as established before, all sections of $L$ vanish along divisors of the form

$$
\bigsqcup_{i=1}^{k}\left(B \times y_{i}\right) \cup f \text {, for } y_{1}+\cdots+y_{k}=o_{F} .
$$

A zero dimensional subscheme $I_{Z}$ in $X_{F}^{[k]}$ consisting of distinct points $z_{i}=\left(x_{i}, y_{i}\right) \in$ $B \times F, 1 \leq i \leq k$, must satisfy

$$
x_{1}+\cdots+x_{k}=o_{B} .
$$

Choose $Z$ so that $y_{1}+\cdots+y_{k} \neq o_{F}$, with $y_{i} \neq y_{j}$ for $i \neq j$, and so that $x_{i} \neq o_{B}$ for all $1 \leq i \leq k$. Then no section of $L$ vanishes at $Z$.

To carry out the induction, note the exact sequence on $X$

$$
0 \rightarrow L_{k, n} \rightarrow L_{k, n+1} \rightarrow \mathcal{O}_{f}\left(k o_{F}\right) \rightarrow 0,
$$

and the associated exact sequence on global sections,

$$
0 \rightarrow H^{0}\left(X, L_{k, n}\right) \rightarrow H^{0}\left(X, L_{k, n+1}\right) \rightarrow H^{0}\left(X, \mathcal{O}_{f}\left(k o_{F}\right)\right) \rightarrow 0 .
$$

Let $I_{Z} \in X_{F}^{[k n]}$ be so that

$$
H^{0}\left(I_{Z} \otimes L_{k, n}\right)=0 \text { and } Z \cap\left(o_{B} \times F\right)=\emptyset .
$$

Then $H^{1}\left(I_{Z} \otimes L_{k, n}\right)=0$. Choose $k$ additional points

$$
z_{i}=\left(o_{B}, y_{i}\right), 1 \leq i \leq k, \text { so that } y_{1}+\cdots y_{k} \neq o_{F} .
$$

From the exact sequence on global sections, it follows that no section of $L_{k, n+1}$ vanishes at

$$
Z \cup\left\{z_{1}, \ldots, z_{k}\right\} \in X_{F}^{[k(n+1)]} .
$$

Indeed, the exact sequence and the induction hypothesis on $Z$ show that

$$
H^{0}\left(X, L_{k, n+1} \otimes I_{Z}\right) \cong H^{0}\left(F, \mathcal{O}_{F}\left(k o_{F}\right) \otimes I_{Z}\right) \cong H^{0}\left(F, \mathcal{O}_{F}\left(k o_{F}\right)\right)
$$

via restriction. But no section of $\mathcal{O}_{F}\left(k o_{F}\right)$ on the central fiber vanishes at the points $z_{1}, \ldots, z_{k}$, so no section of $L_{k, n+1}$ vanishes at $Z \cup\left\{z_{1}, \ldots, z_{k}\right\}$. We conclude that for a general $I_{Z^{\prime}} \in X_{F}^{[k(n+1)]}$, we have

$$
H^{0}\left(I_{Z^{\prime}} \otimes L_{k, n+1}\right)=0 .
$$

This ends the proof of the lemma. 
4.4. Proof of Theorem 2A. The strategy of proof is similar to that of Theorem 2, We indicate the necessary changes. We write

$$
\operatorname{det} v=\mathcal{O}\left(\sigma+m_{v} f\right) \otimes Q_{v}^{-r}, \operatorname{det} w=\mathcal{O}\left(\sigma+m_{w} f\right) \otimes Q_{w}^{-s},
$$

for line bundles $Q_{v}, Q_{w}$ of degree 0 over $C$. We recall the birational isomorphism in Remark 3.1

$$
\mathfrak{M}_{v}^{+--\rightarrow} \mathfrak{X}_{v}^{+}=\left\{\left(Z, z_{C}\right): z_{C}^{r}=\mathcal{M}_{Z}\right\} \subset X^{\left[d_{v}\right]} \times \operatorname{Pic}^{0}(C)
$$

where we set

$$
\mathcal{M}_{Z}=\mathcal{O}_{C}\left(a_{C}(Z)-d_{v} \cdot o\right) .
$$

This was noted when $Q_{v}$ is trivial in Section 3.2, but the twist by $Q_{v}$ is an isomorphism of moduli spaces, yielding only a modified formula

$$
\mathbf{R} \mathcal{S}^{\dagger}(V)=I_{Z}^{\vee}(-r \sigma+(\chi+\bar{g}) f) \otimes z_{C}^{-1} \otimes Q_{v}^{-1} .
$$

There is a similar birational isomorphism $\mathfrak{M}_{w}^{+}-\rightarrow \mathfrak{X}_{w}^{+}$.

The divisor $\Theta_{v w}^{+} \subset \mathfrak{M}_{v}^{+} \times \mathfrak{M}_{w}^{+}$is identified with

$$
\Theta^{+} \subset \mathfrak{X}_{v}^{+} \times \mathfrak{X}_{w}^{+}
$$

where

$$
\Theta^{+}=\left\{\left(Z, T, z_{C}, t_{C}\right): h^{0}\left(I_{Z} \otimes I_{T} \otimes z_{C} \otimes t_{C} \otimes L\right) \neq 0\right\}
$$

for the line bundle

$$
L=\mathcal{O}\left((r+s) \sigma-\left(\chi_{v}+\chi_{w}+2 \bar{g}\right) f\right) \otimes Q \Longrightarrow \chi(L)=d_{v}+d_{w}
$$

Here, we wrote

$$
Q=Q_{v} \otimes Q_{w}
$$

which by assumption is a generic line bundle of degree 0 over the curve $C$. It can be seen that $L$ has no higher cohomology if $\chi_{v}+\chi_{w} \leq-3 \bar{g}$. In turn, when $r=s$, this is equivalent to the requirement

$$
d_{v}+d_{w}=-2 r\left(\chi_{v}+\chi_{w}+\bar{g}\right) \geq 4 r \bar{g}
$$

of the theorem.

There are a few steps in the proof of Theorem $2 \mathrm{~A}$ that need modifications from the genus 1 case. They are:

(i) the identification of the theta bundles. We carry this out for $r=s$ only, making use of the natural addition map

$$
\tau^{+}: \mathfrak{X}_{v}^{+} \times \mathfrak{X}_{w}^{+}-\rightarrow \mathfrak{X}^{+},
$$


where

$$
\mathfrak{X}^{+}=\left\{\left(U, u_{C}\right): \mathcal{M}_{U}=u_{C}^{r}\right\} \subset X^{\left[d_{v}+d_{w}\right]} \times \operatorname{Pic}^{0}(C) .
$$

We have $\Theta^{+}=\left(\tau^{+}\right)^{\star} \theta^{+}$, for

$$
\theta^{+}=\left\{\left(U, u_{C}\right): h^{0}\left(I_{U} \otimes u_{C} \otimes L\right) \neq 0\right\} .
$$

As before, we note the natural projections

$$
c: \mathfrak{X}^{+} \rightarrow X^{\left[d_{v}+d_{w}\right]}, \operatorname{pr}: \mathfrak{X}^{+} \rightarrow \operatorname{Pic}^{0}(C) .
$$

We claim that

$$
\mathcal{O}\left(\theta^{+}\right)=c^{\star} L^{\left[d_{v}+d_{w}\right]} \otimes \operatorname{pr}^{\star} \mathcal{P}_{\alpha}^{r},
$$

where $\mathcal{P}_{\alpha}$ is the line bundle over $\operatorname{Pic}^{0}(C)$ associated to the point

$$
\alpha=K_{C}(-2 \bar{g} \cdot o) \otimes Q^{-2} \in \operatorname{Pic}^{0}(C) .
$$

Pulling back under $\tau^{+}$, it follows that

$$
\Theta_{w}=c_{v}^{\star} L^{\left[d_{v}\right]} \otimes \operatorname{pr}^{\star} \mathcal{P}_{\alpha}^{r}, \Theta_{v}=c_{w}^{\star} L^{\left[d_{w}\right]} \otimes \operatorname{pr}^{\star} \mathcal{P}_{\alpha}^{r} .
$$

Before proving (16), we simplify notations. We write

$$
\ell=\chi(L)=d_{v}+d_{w} .
$$

Over the Jacobian

$$
A=\operatorname{Pic}^{0}(C),
$$

we fix a principal polarization, for instance

$$
\Theta=\left\{y \in A: h^{0}\left(y \otimes \mathcal{O}_{C}(\bar{g} \cdot o)\right) \neq 0\right\} .
$$

The standardly normalized Poincaré bundle

$$
\mathcal{P} \rightarrow A \times A
$$

takes the form

$$
\mathcal{P}=m^{\star} \Theta^{-1} \otimes(\Theta \otimes \Theta) .
$$

This differs up to a sign from the usual conventions, but it is compatible with our conventions on the Abel-Jacobi embedding

$$
C \rightarrow A, x \rightarrow \mathcal{O}_{C}(x-o),
$$

in the sense that that $\left.\mathcal{P}\right|_{A \times \alpha}$ restricts to $\alpha$ over $C$. For degree $\ell$, we consider the Abel-Jacobi map

$$
\pi: C^{(\ell)} \rightarrow A, D \mapsto \mathcal{O}(D-\ell \cdot o),
$$


and set

$$
\mathcal{P}_{C}^{(\ell)}=(\pi, 1)^{\star} \mathcal{P} \rightarrow C^{(\ell)} \times A .
$$

The bundle $\mathcal{P}^{(\ell)}$ satisfies

$$
\left.\mathcal{P}^{(\ell)}\right|_{C^{(\ell)} \times\{y\}} \cong y^{(\ell)},\left.\mathcal{P}^{(\ell)}\right|_{\ell[o] \times A} \text { is trivial over } A .
$$

When $\ell=1, \mathcal{P}^{(1)}$ is the Poincaré bundle

$$
\mathcal{P}_{C} \rightarrow C \times A
$$

normalized over $o$.

With these notations out of the way, we first consider $\mathcal{O}\left(\theta^{+}\right)$over the product $X^{[\ell]} \times A$. We claim that

$$
\mathcal{O}\left(\theta^{+}\right)=c^{\star} L^{[\ell]} \otimes\left(a_{C}, 1\right)^{\star} \mathcal{P}^{(\ell)} \otimes \operatorname{pr}_{A}^{\star} \mathcal{M}
$$

for some line bundle $\mathcal{M} \rightarrow A$. This follows from the see-saw theorem. The restriction of $\mathcal{O}\left(\theta^{+}\right)$to $X^{[\ell]} \times\left\{u_{C}\right\}$ is

$$
\left(L \otimes u_{C}\right)^{[\ell]}=L^{[\ell]} \otimes a_{C}^{\star} u_{C}^{(\ell)} .
$$

This agrees with the restriction of

$$
c^{\star} L^{[\ell]} \otimes\left(a_{C}, 1\right)^{\star} \mathcal{P}^{(\ell)}
$$

and establishes (18). To identify $\mathcal{M}$, we restrict to $\{U\} \times A$, where $U$ is a length $\ell$ subscheme of $X$ supported over $o \times o_{F}$. We obtain

$$
\mathcal{M}=\operatorname{det} \mathbf{R} p_{!}\left(\mathcal{P}_{C} \otimes q^{\star}\left(L \otimes I_{U}\right)\right)^{-1} .
$$

We can rewrite $\mathcal{M}$ expressing in $K$-theory

$$
I_{U}=\mathcal{O}-\ell \cdot \mathcal{O}_{o \times o_{F}} .
$$

Recalling the normalization of $\mathcal{P}_{C}$ over $o$, and that

$$
L=\left(\mathcal{O}_{C}(t \cdot o) \otimes Q\right) \otimes \mathcal{O}_{F}\left(2 r \cdot o_{F}\right),
$$

for $t=-\left(\chi_{v}+\chi_{w}+2 \bar{g}\right)$, we obtain

$$
\begin{gathered}
\mathcal{M}=\operatorname{det} \mathbf{R} p_{!}\left(\mathcal{P}_{C} \otimes q^{\star} L\right)^{-1}=\operatorname{det} \mathbf{R} p_{!}\left(\mathcal{P}_{C} \otimes q^{\star}\left(\mathcal{O}_{C}(t \cdot o) \otimes Q\right)\right)^{-2 r} \\
=\operatorname{det} \mathbf{R} p_{!}\left(\mathcal{P}_{C} \otimes q^{\star}\left(\mathcal{O}_{C}(\bar{g} \cdot o) \otimes Q\right)\right)^{-2 r}=\Theta^{2 r} \otimes \mathcal{P}_{-Q}^{2 r} .
\end{gathered}
$$

Therefore

$$
\mathcal{O}\left(\theta^{+}\right)=c^{\star} L^{[\ell]} \otimes\left(a_{C}, 1\right)^{\star} \circ(\pi, 1)^{\star} \mathcal{P} \otimes \operatorname{pr}_{A}^{\star}\left(\Theta \otimes \mathcal{P}_{-Q}\right)^{2 r} .
$$

Over $\mathfrak{X}^{+} \hookrightarrow X^{[\ell]} \times A$, we have the following commutative diagram 


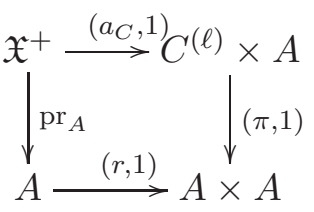

so that

$$
(\pi, 1) \circ\left(a_{C}, 1\right)=(r, 1) \circ \operatorname{pr}_{A} .
$$

Hence, we obtain

$$
\begin{aligned}
\mathcal{O}\left(\theta^{+}\right) & =c^{\star} L^{[\ell]} \otimes \operatorname{pr}_{A}^{\star}\left((r, 1)^{\star} \mathcal{P} \otimes \Theta^{2 r} \otimes \mathcal{P}_{-Q}^{2 r}\right) \\
& =c^{\star} L^{[\ell]} \otimes \operatorname{pr}_{A}^{\star}\left(r^{\star} \Theta \otimes \Theta \otimes(r+1)^{\star} \Theta^{-1} \otimes \Theta^{2 r} \otimes \mathcal{P}_{-Q}^{2 r}\right) \\
& =c^{\star} L^{[\ell]} \otimes \operatorname{pr}_{A}^{\star}\left(\Theta \otimes(-1)^{\star} \Theta^{-1} \otimes \mathcal{P}_{-Q}^{2}\right)^{r}=c^{\star} L^{[\ell]} \otimes \operatorname{pr}_{A}^{\star} \mathcal{P}_{\alpha}^{r},
\end{aligned}
$$

as claimed.

(ii) Next, we identify the space of sections $H^{0}\left(\mathfrak{X}^{+}, \mathcal{O}\left(\theta^{+}\right)\right)$using the cartesian diagram

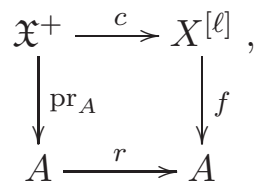

where $f=\pi \circ a_{C}$. We have

$$
\begin{aligned}
H^{0}\left(\mathfrak{X}^{+}, \mathcal{O}\left(\theta^{+}\right)\right) & =H^{0}\left(\mathfrak{X}^{+}, c^{\star} L^{[\ell]} \otimes \operatorname{pr}_{A}^{\star} \mathcal{P}_{\alpha}^{r}\right)=H^{0}\left(X^{[\ell]}, L^{[\ell]} \otimes c_{\star} \operatorname{pr}_{A}^{\star} \mathcal{P}_{\alpha}^{r}\right) \\
& =H^{0}\left(X^{[\ell]}, L^{[\ell]} \otimes f^{\star} r_{\star} \mathcal{P}_{\alpha}^{r}\right)=H^{0}\left(X^{[\ell]}, L^{[\ell]} \otimes f^{\star} r_{\star} r^{\star} \mathcal{P}_{\alpha}\right) \\
& =\bigoplus_{\tau \in A[r]} H^{0}\left(X^{[\ell]}, L^{[\ell]} \otimes f^{\star}\left(\mathcal{P}_{\alpha} \otimes \tau\right)\right) \\
& =\bigoplus_{\tau \in A[r]} H^{0}\left(X^{[\ell]}, L^{[\ell]} \otimes a_{C}^{\star}(\alpha \otimes \tau)\right) \\
& =\bigoplus_{\tau \in A[r]} H^{0}\left(X^{[\ell]},(L \otimes \alpha \otimes \tau)^{[\ell]}\right) .
\end{aligned}
$$

Similar expressions hold over each of the factors $\mathfrak{X}_{v}^{+}$and $\mathfrak{X}_{w}^{+}$.

(iii) Finally, we need a suitable analogue of Lemma 4. This concerns subschemes $Z$ in $X$, belonging to

$$
X_{F}^{[\ell]}=\left\{Z: a_{C}(Z) \text { is rationally equivalent to } \ell \cdot o\right\} .
$$

We show that if

$$
L_{k, n}=\mathcal{O}(k \sigma+n f)
$$

for $k \geq 2 g, n \geq g$, then for all $r$-torsion line bundles $\tau$ over $C$, we have

$$
H^{0}\left(L_{k, n} \otimes \alpha \otimes \tau \otimes I_{Z}\right)=0
$$


provided $Z$ is generic in $X_{F}^{[k(n-\bar{g})]}$, and $Q$, which appears in the definition of $\alpha$ in (17), is generic over $C$. This is then applied to the line bundle $L$ appearing in the expression of the theta bundles above.

We induct on $n$, starting with the base case $n=g$. Just as in genus 1 , for generic $Q$, the sections of $L_{k, g} \otimes \alpha \otimes \tau$ vanish along divisors of the form

$$
\bigsqcup_{i=1}^{k}\left(C \times y_{i}\right) \cup f_{p_{1}}+\ldots+f_{p_{g}} \text {, for } y_{1}+\cdots+y_{k}=o_{F},
$$

and some $p_{1}, \ldots, p_{g} \in C$. Indeed, it suffices to explain that

$$
h^{0}\left(\mathcal{O}_{C}(g \cdot o) \otimes \alpha \otimes \tau\right)=1,
$$

the unique section vanishing at $g$ points $p_{1}(o), \ldots, p_{g}(o)$, which depend on $o$. Equivalently, recalling the definition of $\alpha$ in (17), and using Riemann-Roch and Serre-duality, we prove that

$$
h^{0}\left(\tau^{-1} \otimes \mathcal{O}_{C}((g-2) \cdot o) \otimes Q^{2}\right)=0 .
$$

As the Euler characteristic of the above line bundle is -1 , the space of sections vanishes for generic $Q$ of degree 0 .

To complete the proof of the base case, fix a generic $Q$ as above. It suffices to explain that for all $k \geq 2 g$, we can find $x_{1}, \ldots, x_{k} \in C$ such that

$$
x_{1}+\ldots+x_{k} \equiv k \cdot o, x_{i} \neq p_{j} \text { for all } i, j .
$$

Choosing a section from the non-empty set

$$
H^{0}\left(\mathcal{O}_{C}(k \cdot o)\right)-\bigcup_{j=1}^{g} H^{0}\left(\mathcal{O}_{C}\left(k \cdot o-p_{j}\right)\right)-H^{0}\left(\mathcal{O}_{C}((k-1) \cdot o)\right),
$$

we let $x_{1}, \ldots, x_{k}$ be its zeros. This ends the argument for the base case.

The inductive step does not require any changes from the original Lemma 4. The proof of Theorem $2 \mathrm{~A}$ is now completed.

\section{REFERENCES}

[A] M. F. Atiyah, Vector bundles over an elliptic curve, Proc. London Math. Soc. 7 (1957), 414-452.

[B] T. Bridgeland, Fourier-Mukai transforms for elliptic surfaces, J. Reine Angew. Math. 498 (1998), 115-133.

[BH] M. Bernardara, G. Hein, The Fourier-Mukai algorithm for elliptic surfaces, arXiv:1002.4986.

[F] R. Friedman, Algebraic surfaces and holomorphic vector bundles, Springer, New York, 1998.

[LP] J. Le Potier, Dualité étrange sur le plan projectif, Luminy, December 1996.

[OG] K. O'Grady, The weight-two Hodge structure of moduli spaces of sheaves on a K3 surface, J. Algebraic Geom. 6 (1997), 599-644. 
[MO1] A. Marian, D. Oprea, A tour of theta dualities on moduli spaces of sheaves, Curves and abelian varieties, Contemporary Mathematics, 465, American Mathematical Society, Providence, Rhode Island (2008), 175-202.

[MO2] A. Marian, D. Oprea, Sheaves on abelian surfaces and strange duality, Math. Ann. 343 (2009), $1-33$.

[MOY] A. Marian, D. Oprea, K. Yoshioka, Generic strange duality for K3 surfaces, arXiv:1005.0102.

[M] S. Mukai, Semi-homogeneous vector bundles on an Abelian variety, J. Math. Kyoto Univ. 18 (1978), 239-272.

[O] D. Oprea, Bundles of generalized theta functions over abelian surfaces, arXiv:1106.3890.

[R] S. Ramanan, The moduli spaces of vector bundles over an algebraic curve, Math. Ann. 200 (1973), 69-84.

[Y] K. Yoshioka, Moduli spaces of stable sheaves on abelian surfaces, Math. Ann. 321 (2001), 817-884.

Department of Mathematics

NortheASTERN UNIVERSITY

E-mail address: a.marian@neu.edu

Department of Mathematics

University of California, San Diego

E-mail address: doprea@math.ucsd.edu 